\title{
TRPC1 and TRPC6 Channels Cooperate with TRPV4 to Mediate Mechanical Hyperalgesia and Nociceptor Sensitization
}

\author{
Nicole Alessandri-Haber, Olayinka A. Dina, Xiaoje Chen, and Jon D. Levine \\ Departments of Oral and Maxillofacial Surgery and Medicine and Division of Neuroscience, University of California, San Francisco, San Francisco, \\ California 94143-0440
}

The transient receptor potential vanilloid 4 (TRPV4) contributes to mechanical hyperalgesia of diverse etiologies, presumably as part of a mechanoreceptor signaling complex (Alessandri-Haber et al., 2008). To investigate the hypothesis that a functional interaction between TRPV4 and stretch-activated ion channels (SACs) is involved in this mechanical transduction mechanism, we used a selective SACs inhibitor, GsMTx-4. Intradermal injection of GsMTx-4 in the rat hindpaw reversed the mechanical hyperalgesia induced by intradermal injection of inflammatory mediators. In vivo single fiber recordings showed that GsMTx-4 reversed inflammatory mediator-induced decrease in mechanical threshold in half of sensitized C-fibers. Furthermore, GsMTx-4 reduced hyperalgesia to both mechanical and hypotonic stimuli in different models of inflammatory and neuropathic pain, although it had no effect on baseline mechanical nociceptive thresholds.

TRPC1 and TRPC6, two GsMTx-4-sensitive SACs, are expressed in dorsal root ganglion (DRG) neurons. Single-cell reverse transcription-PCR showed that messenger RNAs for TRPV4, TRPC1, and TRPC6 are frequently coexpressed in DRG neurons. Spinal intrathecal administration of oligodeoxynucleotides antisense to TRPC1 and TRPC6, like that to TRPV4, reversed the hyperalgesia to mechanical and hypotonic stimuli induced by inflammatory mediators without affecting baseline mechanical nociceptive threshold. However, antisense to TRPC6, but not to TRPC1, reversed the mechanical hyperalgesia induced by a thermal injury or the TRPV4selective agonist $4 \alpha$-PDD ( $4 \alpha$-phorbol 12,13-didecanoate). We conclude that TRPC1 and TRPC6 channels cooperate with TRPV4 channels to mediate mechanical hyperalgesia and primary afferent nociceptor sensitization, although they may have distinctive roles.

\section{Introduction}

The growing number of probable mechanical transducers (Lumpkin and Caterina, 2007) and the emergence of new models of mechanical gating such as the "tensegrity model" (Ingber, 1997, 2003a,b) suggest that the transduction of mechanical stimuli in primary afferent nociceptors depends on the activation of mechanoreceptor signaling complexes rather than a simple stretch-activated mechanotransducer.

The transient receptor potential vanilloid 4 (TRPV4) plays a major role in the mechanical hyperalgesia associated with pronociceptive inflammatory mediators and small-fiber painful peripheral neuropathies (Alessandri-Haber et al., 2004, 2006, 2008). Although TRPV4 does not appear to be gated by mechanical stretch (Strotmann et al., 2000) and does not contribute to baseline mechanical nociceptive threshold (Alessandri-Haber et al., 2003; Liedtke and Friedman, 2003; Suzuki et al., 2003), it does

Received Feb. 21, 2009; accepted March 29, 2009.

This work was supported by a grant from the National Institutes of Health. We thank Dr. Oliver Bogen for his helpful comments on this manuscript and Drs. Wolfgang Liedkte and Jeffrey Friedman for kindly providing the TRPV4 knock-out mice.

Correspondence should be addressed to Dr. Nicole Alessandri-Haber, Department of Oral and Maxillofacial Surgery, C-522, Box 0440, University of California, San Francisco, 521 Parnassus Avenue, San Francisco, CA 94143-0440. E-mail: nicole.haber@ucsf.edu.

D01:10.1523/JNEUROSCI.0893-09.2009

Copyright $\odot 2009$ Society for Neuroscience $\quad$ 0270-6474/09/296217-12\$15.00/0 contribute to mechanical transduction in sensitized primary afferent nociceptors by functional coupling with other molecules implicated in mechanotransduction such as integrins and Src tyrosine kinases (Alessandri-Haber et al., 2008).

Although stretch-activated channels (SACs) also participate in the detection of mechanical stimuli in dorsal root ganglion (DRG) neurons (McCarter et al., 1999; Cho et al., 2002; Hu and Lewin, 2006), the use of nonselective blockers has hampered our understanding of their role in mechanotransduction (Hamill and McBride, 1996; Hamill, 2006). Recently, more selective SAC blockers have emerged (Suchyna et al., 2000; Meyers et al., 2003; Drew et al., 2007). One of these, GsMTx-4, a small peptide found in the venom of the Chilean rose tarantula spider Grammostola spatula, specifically blocks cationic SACs (Suchyna et al., 2000; Bowman et al., 2007). Intraperitoneal injection of GsMTx-4 has been shown recently to reduce mechanical hyperalgesia induced by carrageenan or sciatic nerve injury (Park et al., 2008), although it does not inhibit SAC currents in cultured DRG neurons (Drew et al., 2007). GsMTx-4 inhibits two members of the canonical subfamily of TRP channels, TRPC1 and TRPC6, which are expressed in DRG neurons and activated by mechanical and hypotonic stimuli (Maroto et al., 2005; Spassova et al., 2006; Bowman et al., 2007; Elg et al., 2007; Kress et al., 2008). In the present study, we investigated whether SACs, and more specifically 
TRPC1 and TRPC6, may function with TRPV4 to transduce mechanical stimuli in sensitized nociceptors.

We demonstrate that local injection of GsMTx-4, at the site of nociceptive testing, reverses hyperalgesia to mechanical and hypotonic stimuli induced by combinations of inflammatory mediators, carrageenan, or the cancer chemotherapy drug paclitaxel, without affecting baseline nociceptive mechanical threshold. Similarly, TRPC1 and TRPC6 participate in the hyperalgesia to mechanical and hypotonic stimuli induced by inflammatory mediators without contributing to baseline nociceptive mechanical threshold. We suggest that TRPC6 and TRPC1 contribute, with TRPV4, to a mechanism mediating primary afferent nociceptor sensitization and mechanical hyperalgesia.

\section{Materials and Methods Animals}

Experiments were performed on 180-200 g adult male Sprague Dawley rats (Charles River) and on male C57BL/6 mice lacking functional TRPV4 gene (TRPV4 ${ }^{-1-}$ mice) (Liedtke and Friedman, 2003) and male TRPV4 wild-type littermates (TRPV4 ${ }^{+/+}$mice). The genotype of the mice was confirmed by PCR of tail DNA. Experimental protocols were approved by the University of California, San Francisco Committee on Animal Research and conformed to National Institutes of Health guidelines for the use of animals in research.

\section{Drugs}

Paclitaxel, carrageenan, prostaglandin $\mathrm{E}_{2}\left(\mathrm{PGE}_{2}\right)$, serotonin (5-HT), histamine, substance $\mathrm{P}$, and $4 \alpha$-phorbol 12,13-didecanoate ( $4 \alpha$-PDD) were purchased from Sigma, bradykinin was purchased from ICN Biomedicals, and GsMTx-4 was from Peptides International.

For behavioral experiments, stock solutions of carrageenan, bradykinin, 5-HT, substance $\mathrm{P}$, and histamine were made in saline. Stock solutions of $\mathrm{PGE}_{2}$ and GsMTx-4 were made in $10 \%$ ethanol and in distilled water, respectively. For all drugs, final experimental dilutions were made in saline on the day of the experiment (final concentrations of ethanol or DMSO were $<1 \%)$.

\section{Pain models}

Carrageenan and inflammatory soup. A solution of either carrageenan ( $1 \% \mathrm{w} / \mathrm{v}, 5 \mu \mathrm{l})$, inflammatory soup $\left(\mathrm{PGE}_{2}, 5-\mathrm{HT}\right.$, histamine, substance $\mathrm{P}$, and bradykinin, $100 \mathrm{ng}$ each, final volume or $2.5 \mu \mathrm{l}$ ) or simplified inflammatory soup ( $\mathrm{PGE}_{2}$ and $5-\mathrm{HT}, 100 \mathrm{ng}$ each, final volume of $2.5 \mu \mathrm{l}$ ) was injected intradermally into the dorsum of the rat hindpaw $30 \mathrm{~min}$ before behavioral testing.

Paclitaxel chemotherapy-induced neuropathy. Paclitaxel was formulated at a concentration of $1 \mathrm{mg} / \mathrm{ml}$ in a vehicle composed of absolute ethanol and Cremophore EL; final paclitaxel concentration of $1 \mu \mathrm{g} / 2.5 \mu \mathrm{l}$ was made in sterile saline at the time of injection (Dina et al., 2001; Alessandri-Haber et al., 2004). Paclitaxel was injected intraperitoneally once a day for $10 \mathrm{~d}$ at a dose of $1 \mathrm{mg} / \mathrm{kg}$. GsMTx-4 was tested on day 11 .

Hindpaw thermal injury. After measuring baseline mechanical thresholds, rats were anesthetized with isoflurane $\left(2.5 \%\right.$ in $\left.97.5 \% \mathrm{O}_{2}\right)$. A brass probe with a surface temperature clamped at $52.5 \pm 0.1^{\circ} \mathrm{C}$ was applied, and pressure between the dorsum of the hindpaw and the probe was maintained with a $9 \mathrm{~g}$ sand pouch for $40 \mathrm{~s}$ (Summer et al., 2006, 2007a). Baseline mechanical threshold testing was performed $24 \mathrm{~h}$ after burn injury.

\section{Mechanical nociceptive threshold}

Mechanical nociceptive thresholds were evaluated by the Randall-Sellito paw-withdrawal test (Ugo-Basile algesymeter; Stoelting) as described previously (Aley and Levine, 2001; Aley et al., 2001). The experiments were not run in a blinded manner. Baseline mechanical thresholds were recorded as the mean of three measurements (at 10 min intervals) before pharmacological reagents. As a negative control, we verified that the injection of $10 \%$ DMSO in rat hindpaw had no effect on paw-withdrawal threshold (see Fig. 7A). PGE 2 and 5-HT (100 ng each/2.5 $\mu \mathrm{l}$ ), $4 \alpha$-PDD, or GsMTx- 4 were injected intradermally $30 \mathrm{~min}$ before behavioral testing. For statistical analysis, each paw was considered to be an independent observation.

\section{Flinch test in rats}

As described previously (Zheng and Chen, 2001; Alessandri-Haber et al., 2003, 2006, 2008; Zhang et al., 2003; Houck et al., 2004), rats were acclimated in a clear plastic observation chamber for $30 \mathrm{~min}$. Then they were briefly restrained while $10 \mu$ l of hypotonic solution (deionized water, 17 mOsm) was administered intradermally into the dorsum of the hindpaw via a 30 gauge needle connected to a $100 \mu$ l syringe by polyethylene tubing. Rats were observed immediately after the injection for a $5 \mathrm{~min}$ period, and the number of flinches observed was recorded as the flinch score. $\mathrm{PGE}_{2}$ and 5-HT (100 ng each/2.5 $\left.\mu \mathrm{l}\right), 4-\alpha \mathrm{PDD}$, or GsMTx-4 were injected intradermally $45 \mathrm{~min}$ before administration of the hypotonic stimulus at the same site of injection. For the experiments testing the recovery from the acute effect of GsMTx-4, flinching and mechanical thresholds were measured $24 \mathrm{~h}$ after the injection of the drugs. For the experiments testing the recovery from the oligodeoxynucleotide (ODN) treatment, flinching and mechanical thresholds were measured $4 \mathrm{~d}$ after the last ODN injection; we showed previously that this time frame was compatible with recovery of the expression level of TRPV4 protein (Alessandri-Haber et al., 2004). A control group of rats was run in parallel to confirm that repeated testing did not affect the mechanical nociceptive threshold or the number of flinches in response to intradermal injection of a hypotonic solution (data not shown).

\section{Oligodeoxynucleotide preparation and administration}

The TRPC1 antisense ODN sequence (5'-TTCTCCTCCTTCACCTCTCG-3') and the TRPC6 antisense ODN sequence (5'- ATAGTCCTGGCTCTCGTTGC-3') (Invitrogen) were directed against a unique region of the rat TRPC1 or TRPC6 channels (GenBank accession numbers, respectively, NM-053558 and NM-053559). The mismatch ODN sequence was designed by mismatching 7 and 8 bases (denoted by boldface) of the TRPC1 antisense (5'-AACTCCTGGTTCAGCAGTCG- ${ }^{\prime}$ ) and TRPC6 antisense ( $5^{\prime}$-TATCTCCTCGCTCTCCAAGC-3'), respectively.

ODN was reconstituted in nuclease-free $0.9 \% \mathrm{NaCl}(10 \mu \mathrm{g} / \mu \mathrm{l})$ and was administered into the spinal intrathecal space at a dose of $40 \mu \mathrm{g} / 20$ $\mu \mathrm{l}$, once a day for $3 \mathrm{~d}$. For this procedure, rats were anesthetized with $2.5 \%$ isoflurane $\left(97.5 \% \mathrm{O}_{2}\right)$, a 30 gauge needle was inserted into the subarachnoid space on the midline between L4 and L5 vertebrae, and ODN was injected at $1 \mu \mathrm{l} / \mathrm{s}$ by microsyringe (Alessandri-Haber et al., 2003).

\section{Antibodies}

The monoclonal anti-TRPC1 [EP1417Y] antibody (ab51255) generated in rabbit against the epitope KFHDFADRKDWDAFC, corresponding to residues 496-509 of human TRPC1 (GenBank accession number I38361) was purchased from Abcam. To verify the specificity of this antibody, we tested in parallel the anti-TRPC1 antibody from Alomone Labs (ACC-010), the selectivity of which has been demonstrated previously (Kim et al., 2003; Liu et al., 2008). In addition, we could show a significant decrease in the level of TRPC1 protein after treatment with TRPC1 antisense ODN, whereas the level of TRPC1 protein was not affected by treatment with TRPC6 antisense ODN (data not shown).

The polyclonal anti-TRPC6 antibody (ACC-017) generated in rabbit against the epitope RRNESQDYLLMDELG, corresponding to residues 24-38 of mouse TRPC6 (GenBank accession number Q61143), was purchased from Alomone Labs. The selectivity of this antibody has been shown previously (Strübing et al., 2003; Dalrymple et al., 2004; Kress et al., 2008; Liu et al., 2008). We confirmed the specificity of this antibody by testing in parallel the anti-TRPC6 antibody from Abcam (ab47679). Furthermore, we could show a significant decrease in the level of TRPC6 protein after treatment with TRPC6 antisense ODN, whereas the level of TRPC6 protein remained unaffected by treatment with TRPC1 antisense ODN (data not shown).

\section{Western blot}

To confirm that the reduction in the nociceptive behaviors associated with ODN treatment resulted from a decrease in the level of TRPC1 and TRPC6 protein, lumbar DRG neurons (L5-L6) from ODN-treated rats were harvested $24 \mathrm{~h}$ after the last ODN injection. Membrane protein preparation and Western blot analyses were performed as described previously (Alessandri-Haber et al., 2003). Briefly, protein concentration for each sample was determined by the bicinchoninic acid (BCA) method 
using the MICRO BCA protein assay kit (Pierce); proteins were loaded on a polyacrylamide gel ( $30 \mu \mathrm{g} / \mathrm{lane})$ and separated by electrophoresis. The membrane was probed with affinity-purified anti-TRPC1 (1:500; Abcam) or anti-TRPC6 antibody (1:500; Alomone Labs), followed by incubation with horseradish peroxidase (HRP)-conjugated goat antirabbit IgG (1:5000; Pierce). To normalize the loaded samples, affinitypurified mouse monoclonal anti-glyceraldehyde-3-phosphate dehydrogenase (GAPDH) antibody (1:5000; Abcam) was used, followed by incubation with HRP-conjugated goat anti-mouse IgG (1:5000; Pierce). Membranes were incubated with enhanced chemiluminescence reagents (Pierce), and images of the membrane were acquired with the CHEMILMAGER chemiluminescence imaging system and analyzed with AlphaEaseFC software (Alpha Innotech). The density of the band of interest (TRPC1 or TRPC6) was measured and normalized to the density of the band of GAPDH. The percentage decrease in level of expression of the protein was then calculated using the following formula: ((average of normalized density for antisense - average of normalized density for mismatch)/average of normalized density for mismatch) ${ }^{\star} 100$. The SEM was calculated with the following formula: (SEM of average of normalized density for antisense/average of normalized density for mismatch) ${ }^{\star} 100$.

\section{DRG membrane preparation, subcellular fractionation}

Lumbar DRG (L4-L6) neurons isolated from adult male rats and cultured for $2 \mathrm{~d}$ were stimulated with a hypotonic solution containing $\mathrm{PGE}_{2}$ and 5-HT (10 $\mu \mathrm{M}$ each) for $15 \mathrm{~min}$. Cells were then homogenized in homogenization buffer [10 mM HEPES, 1 mM EDTA, $0.320 \mathrm{~m}$ sucrose, and complete cocktail of protease inhibitors (Roche), $\mathrm{pH}$ 7.4]. The homogenate was centrifuged at $1000 \times g$ for $10 \mathrm{~min}$ at $4^{\circ} \mathrm{C}$. The supernatant was placed on ice, and the pellet was resuspended in homogenization buffer. This step was repeated twice. Then, supernatants were pooled and centrifuged at $15,000 \times g$ for $25 \mathrm{~min}$ at $4^{\circ} \mathrm{C}$. The pellet was resuspended in $5 \mathrm{~mm}$ Tris- $\mathrm{HCl}, 0.320 \mathrm{~m}$ sucrose, and cocktail of proteases inhibitors, $\mathrm{pH}$ 8.1, layered on $5 \mathrm{~mm}$ Tris- $\mathrm{HCl}$ solution containing $1.2 \mathrm{~m}$ sucrose, and centrifuged at $35,000 \times g$ for $1 \mathrm{~h}$ at $4^{\circ} \mathrm{C}$. The membrane fraction was diluted to a final sucrose concentration $<0.3 \mathrm{~m}$ and centrifuged at $20,000 \times g$ for $90 \mathrm{~min}$ at $4^{\circ} \mathrm{C}$. Protein concentration was estimated by spectrophotometry at $280 \mathrm{~nm}$. Protein samples were separated by PAGE, and Western blot analysis was performed. The membrane was probed in parallel with affinity-purified anti-TRPC1 (1:500; Abcam), anti-TRPC6 antibody (1:500; Alomone Labs), or anti-TRPV4 (1:500; Alomone Labs), followed by incubation with HRP-conjugated goat anti-rabbit IgG (1: 5000 ; Pierce).

\section{In vivo single fiber electrophysiology}

Rats were anesthetized with sodium pentobarbital (initially $50 \mathrm{mg} / \mathrm{kg}$, i.p.), with additional doses given throughout the experiment to maintain areflexia. Single C-fibers from the saphenous nerve, with cutaneous receptive fields, were recorded as described previously (Chen and Levine, 1999). Briefly, recordings were made from the saphenous nerve, which innervates the mediodorsal surface of the hindpaw. Bipolar stimulating electrodes were placed under the nerve at a site distal to the recording site. The nerve was crushed proximal to the recording site to prevent flexor reflexes during electrical stimulation of the nerve. Fine fascicles of axons were dissected from the nerve and placed on a silver-wire recording electrode. Single units were first detected by electrical stimulation of the nerve. The conduction velocity of a fiber was determined by dividing the distance between the stimulating and recording electrodes by the action potential latency. Fibers with conduction velocities $<2 \mathrm{~m} / \mathrm{s}$ were classified as C-fibers (Willis, 1985). The action potential corresponding to the C-fiber whose receptive field had been identified was determined by the latency delay technique, in which electrically evoked spikes resulted in longer latency when the receptive field of the same fiber was stimulated (Handwerker et al., 1991). Receptive fields of individual C-fibers were located and measured using a mechanical search stimulus. The fiber was determined as cutaneous if it was activated by lifting and stimulating the skin and/or if the receptive fields move when the skin is moved relative to subcutaneous tissue. Noncutaneous fibers were not further studied. Spontaneous activity of C-fibers was measured as the number of action potentials fired during a 2 min period, in the absence of applied stimu- lation. Mechanical threshold was determined with calibrated von Frey hairs (Ainsworth) and defined as the lowest force that elicited two or more spikes within $1 \mathrm{~s}$, in at least 6 of 10 trials. Sustained (60 s) suprathreshold ( $10 \mathrm{~g})$ mechanical stimulation was performed using a mechanical stimulator consisting of a force-measuring transducer (Entran) mounted in series with interchangeable von Frey hair filaments. Once the receptive field was delimited and the response to mechanical stimulus characterized, GsMTx-4 (1000 ng in $2.5 \mu \mathrm{l})$ or the simplified soup ( $\mathrm{PGE}_{2}$ and 5 -HT, $100 \mathrm{ng}$ of each in a final volume of $2.5 \mu \mathrm{l}$ ) were injected intradermally $\sim 1 \mathrm{~mm}$ from the center of the mechanical receptive field of a C-fiber.

\section{Dorsal root ganglion cell culture}

L4-L6 DRG were harvested from TRPV4 $4^{+/+}$and TRPV4 ${ }^{-/-}$mice and dissociated and cultured as described previously (Alessandri-Haber et al., 2003). Briefly, dissociated cells were plated on coverslips treated with poly-DL-ornithine ( $0.1 \mathrm{mg} / \mathrm{ml}$; Sigma) and laminin ( $5 \mu \mathrm{g} / \mathrm{ml}$; Invitrogen) and incubated at $37^{\circ} \mathrm{C}$ in $96.5 \%$ air, $3.5 \% \mathrm{CO}_{2}$. Neurons were maintained in culture for $2 \mathrm{~d}$ in DMEM supplemented with $50-100 \mathrm{ng} / \mathrm{ml}$ nerve growth factor, $100 \mathrm{U} / \mathrm{ml}$ penicillin/streptomycin, MEM vitamins, and $10 \%$ heat-inactivated fetal calf serum (all from Invitrogen).

\section{Calcium imaging}

Calcium imaging was performed using the fluorescent calcium indicator fura-2 AM $48 \mathrm{~h}$ after dissociation, as described previously (AlessandriHaber et al., 2003). Briefly, neurons were loaded with $5 \mu \mathrm{M}$ fura- $2 \mathrm{AM}$ for $20 \mathrm{~min}$ in isotonic solution ( $312 \mathrm{mOsm})$. Experiments were performed at $20-23^{\circ} \mathrm{C}$ with the perfusion at a flow rate of $1-2 \mathrm{ml} / \mathrm{min}$. Cells were perfused with isotonic solution for $10 \mathrm{~min}$ before recordings to allow complete removal of unhydrolyzed fura ester.

Measurement of the concentration of free calcium ions $\left(\left[\mathrm{Ca}^{2+}\right]_{i}\right)$ was performed by ratiometric imaging with an intensified charge-coupled device camera. Fluorescence was excited at 340 and $380 \mathrm{~nm}$, and emitted light was long filtered at $510 \mathrm{~nm}$. The fluorescence ratio, $F_{340} / F_{380}$, was calculated with METAFLUOR software (Molecular Devices). Calcium calibration was performed with a fura- 2 calcium imaging calibration kit (Invitrogen), and apparent free $\left[\mathrm{Ca}^{2+}\right]_{\mathrm{i}}$ was calculated from the equation $\left[\mathrm{Ca}^{2+}\right]_{\mathrm{i}}=K_{\mathrm{d}} \times\left(\left(R-R_{\min }\right) /\left(R_{\max }-R\right)\right) \times\left(F_{\max }^{380} / F_{\text {min }}^{380}\right)$, where $R$ is the ratio of $510 \mathrm{~nm}$ emission intensity, exciting at $340 \mathrm{~nm}$, to $510 \mathrm{~nm}$ emission intensity, exciting at $380 \mathrm{~nm}, R_{\min }$ is the ratio at zero free $\mathrm{Ca}^{2+}$, $R_{\max }$ is the ratio at saturating $\mathrm{Ca}^{2+}$ (e.g., $\left.39 \mu \mathrm{M}\right), F_{380}$ max is the fluorescence intensity, exciting at $380 \mathrm{~nm}$, for zero free $\mathrm{Ca}^{2+}$, and $F_{380} \mathrm{~min}$ is the fluorescence intensity at saturating free $\mathrm{Ca}^{2+}$. The $K_{\mathrm{d}}$ can be determined from the fura-2-containing $\mathrm{Ca}^{2+}$ standards and was calculated by using the utility available on the website of the manufacturer. The free $\mathrm{Ca}^{2+}$ for any experimental sample was then calculated from the corresponding $R$ value. Neurons were challenged for $3 \mathrm{~min}$ with a $30 \%$ hypotonic solution containing $\mathrm{PGE}_{2}$ and 5-HT (10 $\mu \mathrm{M}$ each), washed for 10 min with isotonic solution containing $\mathrm{PGE}_{2}$ and 5-HT, perfused with an isotonic solution containing $\mathrm{PGE}_{2}, 5-\mathrm{HT}$, and GsMTx-4 (500 nM) for $5 \mathrm{~min}$, and then challenged with $30 \%$ hypotonic solution containing $\mathrm{PGE}_{2}, 5-\mathrm{HT}$, and GsMTx- 4 for $3 \mathrm{~min}$. Free $\mathrm{Ca}^{2+}$ was calculated from the normalized fluorescence ratio during a $30 \%$ hypotonic solution containing $\mathrm{PGE}_{2}$, $5-\mathrm{HT}$, and GsMTx- 4 divided by the normalized fluorescence ratio in $30 \%$ hypotonic solution containing $\mathrm{PGE}_{2}$ and 5-HT.

We minimized conductance via other ion channels than TRPV4 by using a combination of room temperature, HEPES buffer, and variation of osmolarity only by modifying D-mannitol concentration (AlessandriHaber et al., 2003). Thus, the standard isotonic solution (312 mOsm) contained the following (in mM): $88 \mathrm{NaCl}, 5 \mathrm{KCl}, 1 \mathrm{MgCl}_{2}, 2.4 \mathrm{CaCl}_{2}, 110$ D-mannitol, 10 and HEPES, buffered at $\mathrm{pH} 7.4$ with $\mathrm{NaOH}$. The hypotonic solution was adjusted to $212 \mathrm{mOsm}$ (30\% hypotonic) by lowering the amount of D-mannitol to $10 \mathrm{~mm}$. Osmolarity and $\mathrm{pH}$ were measured before each experiment. The vehicle for the fura-2 AM, DMSO, at its final working dilution did not induce any response in DRG neurons (data not shown). At the end of each experiment, a short exposure to solution containing $20 \mathrm{~mm} \mathrm{KCl}$ was performed to confirm that all cells studied exhibited electrical excitability that is typical of healthy neurons. 
Single-cell reverse transcription- $P C R$

Single-cell reverse transcription (RT)-PCR experiments were conducted as described previously (Alessandri-Haber et al., 2003). Briefly, cytoplasm was aspirated into patch-clamp electrodes from cultured DRG neurons, and reverse transcription was performed immediately using cells-to-cDNA II kit (Applied Biosystems). Two successive PCR amplifications were performed for 28 and 38 cycles using HotStar Taq Plus DNA Polymerase (Qiagen). The following primer pairs were used for TRPV4 (GenBank accession number NM_022017, nucleotides 1480-1700), TRPC1 (GenBank accession number NM_011643, nucleotides 18432010), and TRPC6 (GenBank accession number NM_013838, nucleotides 1417-1644). Gels were analyzed with AlphaEaseFC software (Alpha Innotech).

\section{Data analysis}

Group data are presented as mean \pm SEM, and comparisons between groups was performed by Student's $t$ test or ANOVA, which, if significant, was followed by Tukey's multiple comparisons post hoc test. Significance was defined as a $p$ value $\leq 0.05$.

\section{Results}

GsMTx-4 reverses mechanical hyperalgesia induced by inflammatory soup or carrageenan

Intradermal injection of the SAC inhibitor GsMTx-4 up to a dose of $1000 \mathrm{ng}$ did not affect the baseline mechanical nociceptive threshold (Fig. $1 \mathrm{~A}$, white bars). In contrast, $1000 \mathrm{ng}$ of GsMTx-4 reversed the decrease in mechanical threshold induced by intradermal injection of inflammatory soup $\left(\mathrm{PGE}_{2}\right.$, bradykinin, substance $\mathrm{P}, 5-\mathrm{HT}$, and histamine, $100 \mathrm{ng}$ each) in rat hindpaw (Fig. $1 B$, white bars).

Inflammatory soup sensitizes primary afferent nociceptors by binding to numerous receptors and modulating ion channels (for review, Millan, 1999). We recently demonstrated that, although single inflammatory mediators are insufficient, a simplified inflammatory soup consisting of $\mathrm{PGE}_{2}$ and $5-\mathrm{HT}$ is sufficient to engage the mechanism of mechanotransduction that is dependent on TRPV4 (Alessandri-Haber et al., 2006). Therefore, we next tested whether GsMTx-4 (1000 ng) inhibits the mechanical hyperalgesia induced by intradermal injection of $\mathrm{PGE}_{2}$ and 5-HT (100 ng of each). Coinjection of $\mathrm{PGE}_{2}$ and 5 -HT induced a $37 \pm$ $2 \%$ decrease in mechanical threshold that was reversed by subsequent intradermal injection of GsMTx-4 at the same site (Fig. 2A) (116 $\pm 2 \mathrm{~g}, n=12$ for baseline; $72 \pm 1 \mathrm{~g}, n=12$ after $\mathrm{PGE}_{2}$ and 5-HT; and $116 \pm 2, n=6$ after GsMTx-4; $p<0.001$, Tukey's post hoc multiple comparison test).

We next assessed the contribution of SACs in a third model of inflammation induced by intradermal injection of carrageenan. The mechanical hyperalgesia induced by carrageenan was also reversed by subsequent injection of GsMTx-4 (Fig. 2B) (118 \pm $1 \mathrm{~g}$ for baseline, $75 \pm 2 \mathrm{~g}$ after carrageenan, and $113 \pm 3 \mathrm{~g}$ after GsMTx- $4 ; n=8$ for each group; $p<0.0001$, Tukey's post hoc multiple comparisons test). Twenty-four hours after administration of GsMTx-4, its effect was no longer detectable; the mechanical nociceptive threshold was not significantly different from its pre-GsMTx- 4 value ( $n=8 ; p>0.05$, Tukey's post hoc multiple comparison test). A control group was run in parallel to confirm that repeated testing did not affect mechanical nociceptive threshold (data not shown).

\section{GsMTx-4 acts on sensitized primary afferent nociceptors}

To investigate whether GsMTx-4 acts on primary afferent nociceptors, we investigated the effect of intradermal injection of GsMTx-4 on the activity of single cutaneous C-fiber nociceptors from the saphenous nerve in vivo. None of the fibers showed spontaneous activity. Intradermal injection of GsMTx-4 (1000
A

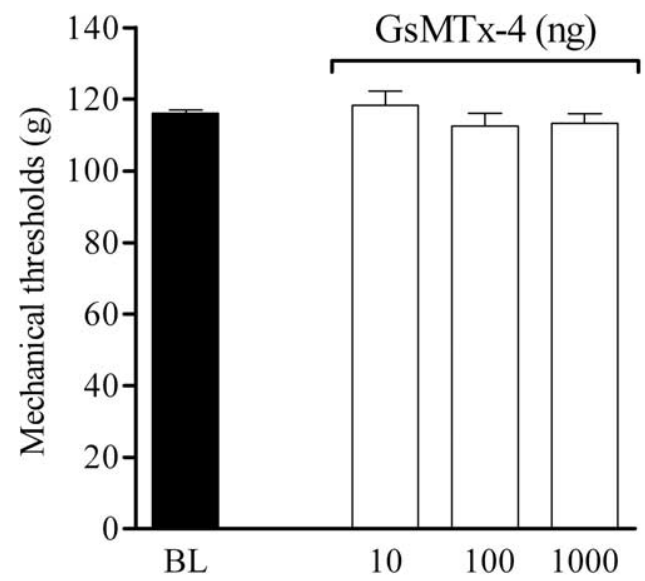

B

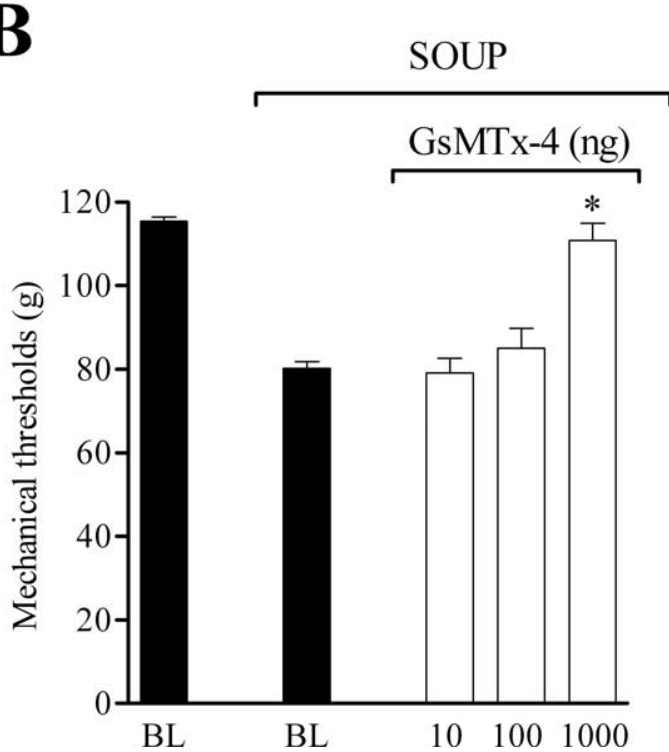

Figure 1. GsMTx-4 inhibits mechanical hyperalgesia without affecting baseline mechanical nociceptive threshold. $\boldsymbol{A}$, Evaluation of nociceptive mechanical threshold in the absence (black bars) or presence (white bars) of different doses of GsMTx-4. Up to a dose of $1000 \mathrm{ng}$, GsMTX-4 did not affect baseline nociceptive mechanical threshold. $\boldsymbol{B}$, Intradermal injection of a soup of inflammatory mediators ( $\mathrm{PGE}_{2}, 5-\mathrm{HT}$, histamine, substance $\mathrm{P}$, and bradykinin, $100 \mathrm{ng}$ each) in rat hindpaw induces a decrease in mechanical nociceptive threshold (black bar). Mechanical nociceptive threshold was evaluated 30 min after administration of different doses of GsMTx-4 (white bars; 10, 100, and $1000 \mathrm{ng}$ ). GsMTx-4 (1000 ng) reversed inflammatory soup-induced mechanical hyperalgesia ( ${ }^{*} p<0.001$, Tukey's post hoc multiple comparison test). BL, Baseline.

ng in $2.5 \mu \mathrm{l})$ did not elicit activity in C-fibers (Fig. 3A) $(n=13)$. Also, GsMTx-4 did not induce any significant change in the average time course of the response of C-fibers to a $60 \mathrm{~s}$ application of a suprathreshold ( $10 \mathrm{~g}$ ) mechanical stimulus (data not shown) nor did it affect the mechanical threshold of these fibers (Fig. $3 A$, inset) ( $n=13 ; p>0.05$, paired Student's $t$ test).

We next investigated the effect of GsMTx- 4 on the response to mechanical stimuli of sensitized cutaneous C-fibers. Exposure to simplified inflammatory soup ( $\mathrm{PGE}_{2}$ and 5-HT, $100 \mathrm{ng}$ of each) induced a decrease in mechanical threshold in 15 of $26 \mathrm{C}$-fibers tested (Fig. 3B) ( $p<0.0003$, paired Student's $t$ test). Subsequent intradermal injection of GsMTx-4 reversed the decrease in mechanical threshold in $50 \%$ of sensitized C-fibers (Fig. $3 B)(p<$ 


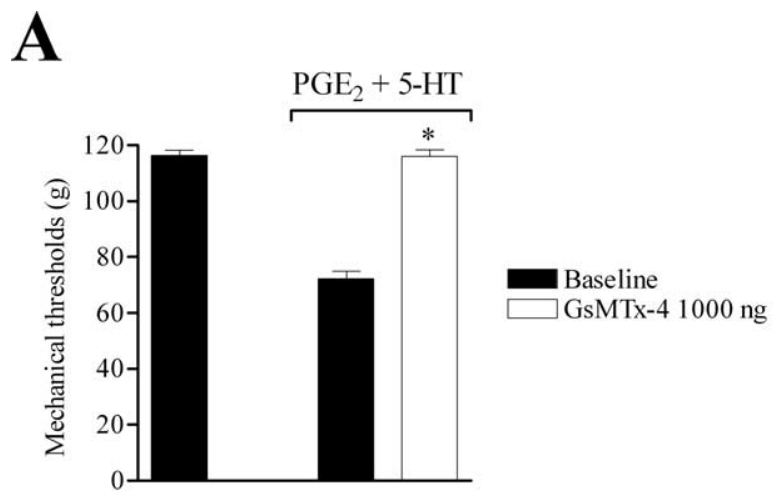

B

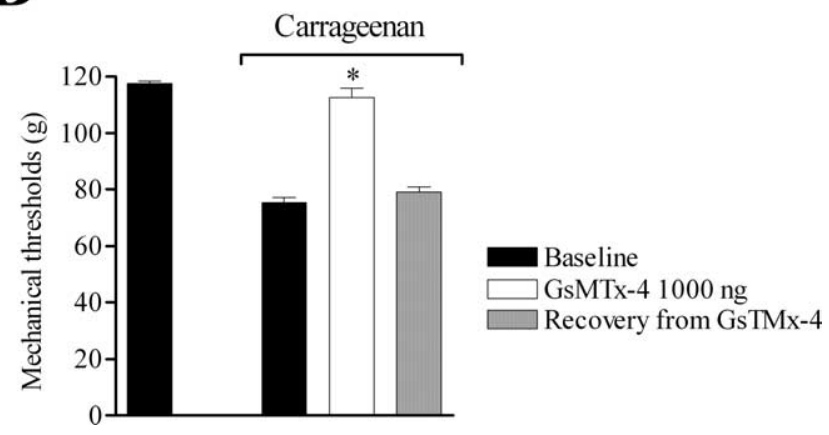

Figure 2. GsMTx-4 reverses mechanical hyperalgesia associated with inflammation. $\boldsymbol{A}$, Intradermal injection of the simplified inflammatory soup ( $\mathrm{PGE}_{2}$ and 5-HT, $100 \mathrm{ng}$ each) induces a decrease in mechanical threshold that was reversed by intradermal injection of GsMTX-4 (1000 $\mathrm{ng}$ ) at the same site ( $116 \pm 2 \mathrm{~g}, n=12$ for baseline; $72 \pm 1 \mathrm{~g}, n=12$ after $\mathrm{PGE}_{2}$ and 5 -HT; and $116 \pm 2 \mathrm{~g}, n=6$ after GsMTx-4; ${ }^{*} p<0.001$, Tukey's post hoc multiple comparison test). $\boldsymbol{B}$, Intradermal injection of carrageenan $(1 \%, 5 \mu \mathrm{l})$ in rat hindpaw induces a significant decrease in mechanical nociceptive threshold that was reversed by intradermal injection of GsMTx-4 (1000 ng) at the same site ( $118 \pm 1 \mathrm{~g}$ for baseline, $75 \pm 2 \mathrm{~g}$ after carrageenan, and $113 \pm 3 \mathrm{~g}$ after GsMTx-4; $n=8 ;{ }^{*} p<0.001$, Tukey's post hoc multiple comparison test). The effect of GsMTx-4 is reversible; $24 \mathrm{~h}$ after its administration, the decrease in mechanical threshold was not significantly different from pre-GsMTx-4 baseline ( $p>$ 0.05 , Tukey's post hoc multiple comparison test).

0.05, $\chi^{2}$ test with Yates correction). To further characterize the subpopulation of sensitized C-fibers that was sensitive to GsMTx-4, we compared the mechanical threshold, conduction velocity, and the number of spikes per minute in response to a sustained mechanical stimulus to those in sensitized C-fibers that were GsMTx-4 insensitive. Based on these criteria, there was no difference between GsMTx-4-sensitive and GsMTx-4-insensitive sensitized C-fibers (Fig. 3B-D) (all $p>0.05$, Tukey's post hoc multiple comparison test). Together with the behavioral experiments, these results suggest that GsMTx-4 inhibits SACs that contribute to mechanical hyperalgesia.

\section{GsMTx-4 reverses paclitaxel-induced} mechanical hyperalgesia

To determine whether SACs also contribute to mechanical hyperalgesia associated with models of painful peripheral neuropathy, we tested the effect of GsMTx-4 on the mechanical hyperalgesia induced by the chemotherapeutic agent paclitaxel. Rats treated with paclitaxel (daily for $10 \mathrm{~d}$ ) develop mechanical hyperalgesia (Fig. 4) (118 $\pm 2 \mathrm{~g}, n=12$ before and $74 \pm 3 \mathrm{~g}, n=6$ after paclitaxel) that lasts for $\sim 2$ weeks (Dina et al., 2001). Intradermal injection of GsMTx-4 in the hindpaw 30 min before testing mechanical nociceptive threshold reversed mechanical hyperalgesia induced by paclitaxel (Fig. 4) (74 \pm 3 g before and $116 \pm 2$ g after
GsMTx-4; $n=6$ for each group; $p<0.001$, Tukey's post hoc multiple comparison test). The effect of GsMTx-4 was reversible, and, $24 \mathrm{~h}$ after its administration, mechanical nociceptive thresholds were not significantly different from pre-GsMTx-4 pawwithdrawal thresholds (Fig. 4) $(n=6$ for each group; $p>0.05$, Tukey's post hoc multiple comparison test).

\section{GsMTx-4 also markedly reduces the sensitization to hypotonic stimuli}

GsMTx-4 inhibits the mechanical hyperalgesia induced by combinations of inflammatory mediators, carrageenan, or paclitaxel to the same extent as spinal intrathecal administration of ODN antisense to TRPV4 (Alessandri-Haber et al., 2004, 2006). Moreover, we demonstrated in different models of inflammation or neuropathy that nocifensive behavior in response to mechanical stimulation (as the total number of paw withdrawals in response to repeated applications of a von Frey hair in the mice and by the Randall-Sellito paw-withdrawal test in the rat) or intradermal injection of hypotonic solution (as the time of licking and shaking of the paw in the mice and the number of flinches in the rat) is absent in rats treated with repetitive intrathecal injection of antisense ODN for TRPV4, or in TRPV4 knockdown mice (Alessandri-Haber et al., 2003, 2005, 2006, 2008). Therefore, to further investigate the effect of GsMTx-4 on TRPV4-dependent nociceptive behavior, we tested whether GsMTx-4 reduces the number of nociceptive flinches in response to a hypotonic solution. Rats treated with inflammatory soup, carrageenan, and paclitaxel have, respectively, a 4-, 3.6-, and 3.2-fold increase in the number of nociceptive flinches in response to an injection of hypotonic solution ( $10 \mu \mathrm{l}$ of deionized water, $17 \mathrm{mOsm})$ in the hindpaw compared with naive rats (Fig. $5 A)(4 \pm 1, n=12$ in naive rats; $16 \pm 2, n=12$ after inflammatory soup; $17 \pm 1, n=12$ in carrageenan-treated rats; and $13 \pm 2, n=10$ in paclitaxeltreated rats; all $p<0.001$, Tukey's post hoc multiple comparison test). As shown in Figure 5A, treatment with GsMTx- 4 reversed the increase in the number of flinches in response to hypotonicity induced by $\mathrm{PGE}_{2}$ and 5-HT, carrageenan, or paclitaxel. Of note, there was no significant difference in the number of flinches between the groups treated with GsMTx-4 and the baseline number of flinches in naive rats (Fig. 5A) (all $p>0.05$, Tukey's multiple comparison test). Again, the effect of the inhibitor was no longer present $24 \mathrm{~h}$ after its administration ( $p>0.05$, Tukey's multiple comparison test). Of note, a control group was run in parallel to confirm that repeated testing did not affect the number of flinches in response to intradermal injection of hypotonic solution (data not shown).

We also demonstrated that TRPV4 functions as an osmotransducer in DRG neurons in vitro (Alessandri-Haber et al., 2003). Moreover, we showed that the increase in concentration of free calcium ions $\left(\left[\mathrm{Ca}^{2+}\right]_{\mathrm{i}}\right)$ induced by a $30 \%$ hypotonic challenge is significantly enhanced in the presence of inflammatory soup in neurons from TRPV4 ${ }^{+/+}$mice but not in neurons from TRPV4 ${ }^{-1-}$ mice (Alessandri-Haber et al., 2006). To further investigate the effect of GsMTx-4 on TRPV4-dependent osmotransduction, we tested the effect of GsMTx-4 on the response of DRG neurons to hypotonic solution in the presence of inflammatory mediators in vitro. Small-diameter DRG neurons $(\leq 25$ $\mu \mathrm{m}$ ) isolated from $T R P V 4^{+/+}$and $T R P V 4^{-/-}$mice were first challenged with a $30 \%$ hypotonic solution containing $\mathrm{PGE}_{2}$ and 5-HT (212 mOsm, $10 \mu \mathrm{M}$ each) for $3 \mathrm{~min}$, and the perfusion was switched to an isotonic solution containing $\mathrm{PGE}_{2}$ and 5-HT (312 $\mathrm{mOsm}$ ) for $10 \mathrm{~min}$. Then the neurons were perfused with isotonic solution containing $\mathrm{PGE}_{2}, 5-\mathrm{HT}$, and GsMTx-4 (500 nM) 

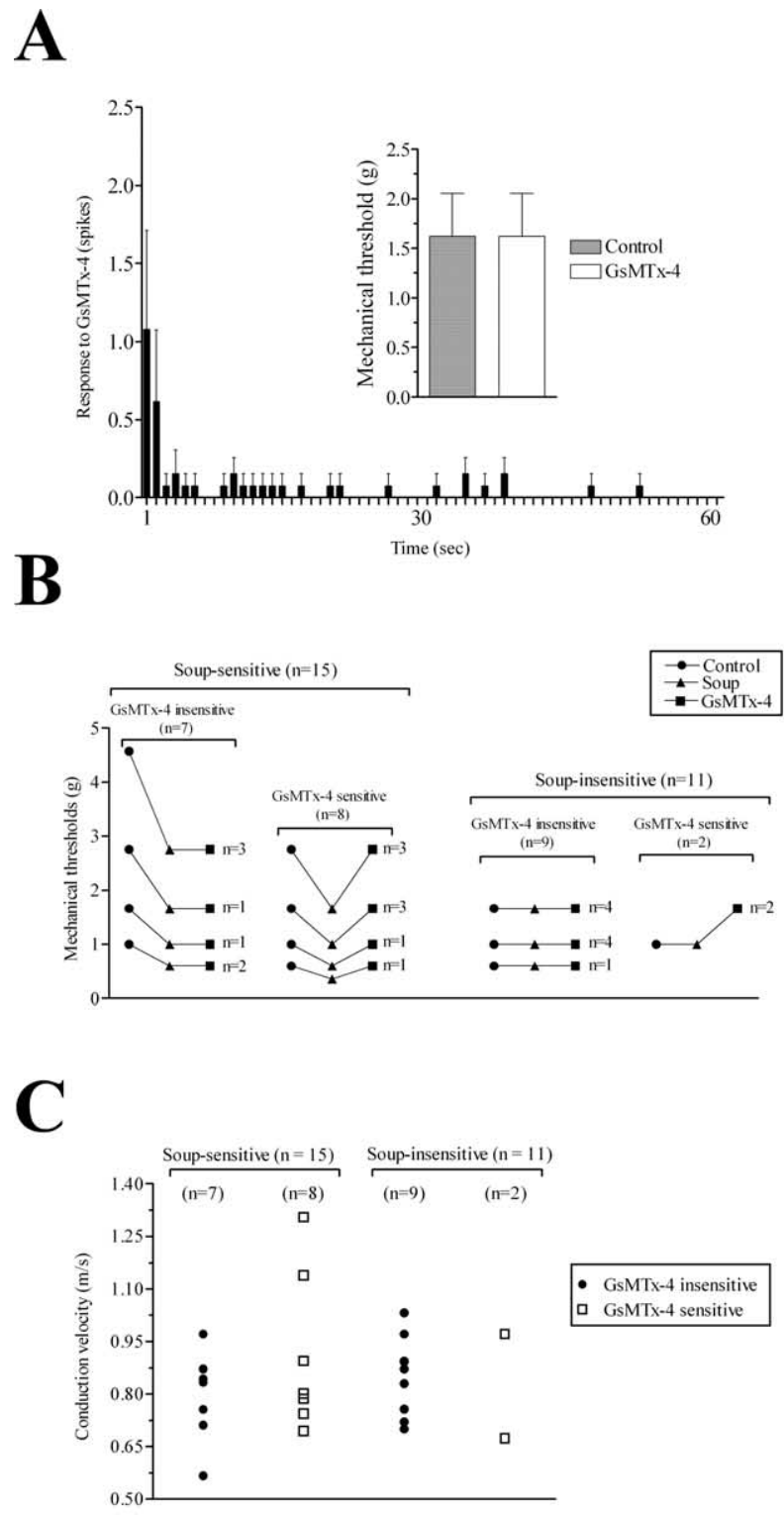

D

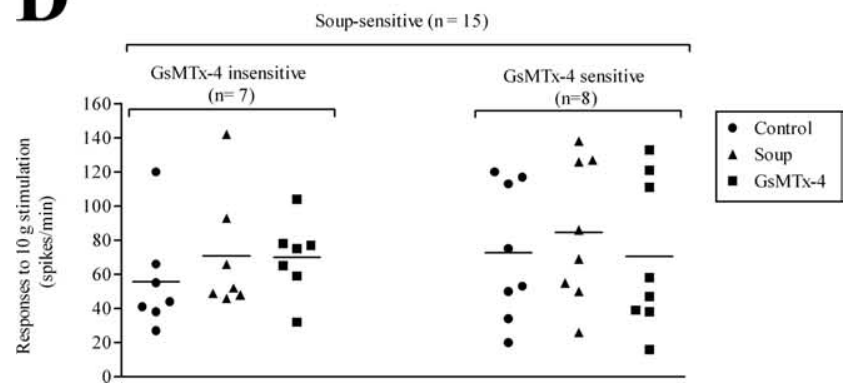

Figure 3. GSMTX-4 reverses the decrease in mechanical threshold induced by inflammatory soup in half of sensitized C-fibers. A, Intradermal injection of GsMTx-4 (1000 ng, $2.5 \mu \mathrm{l}$ ) adjacent to mechanical receptive fields did not induce significant activity in C-fibers. Inset, Mechanical thresholds of $\mathrm{C}-$-fibers were not significantly different from baseline after intradermal injection of GsMTx-4 ( $n=13)$. $\boldsymbol{B}$, Response of individual C-fibers to sustained (60 s) threshold mechanical stimulation before $(\mathbf{O})$ and after $(\mathbf{\Delta})$ intradermal injection of $\mathrm{PGE}_{2}$ and 5 -HT and after a subsequent intradermal injection of GSMTX-4 ( $\boldsymbol{\square})$. $n$ is the number of fibers recorded. Injection of simplified inflammatory soup ( $\mathrm{PGE}_{2}$ and $5-\mathrm{HT}, 100 \mathrm{ng}$ each) into the mechanical receptive field of $\mathrm{C}$-fibers reduced the mechanical threshold in 15 of $26 \mathrm{C}$-fibers. A subsequent injection of GsMTx-4 (1000 ng, $2.5 \mu$ l) into the mechanical receptive field of a C-fiber reversed the decrease in mechanical thresholds induced by simplified soup in $50 \%$ of the sensitized C-fibers (8 of 15). C, There is no significant difference in the conduction velocity between

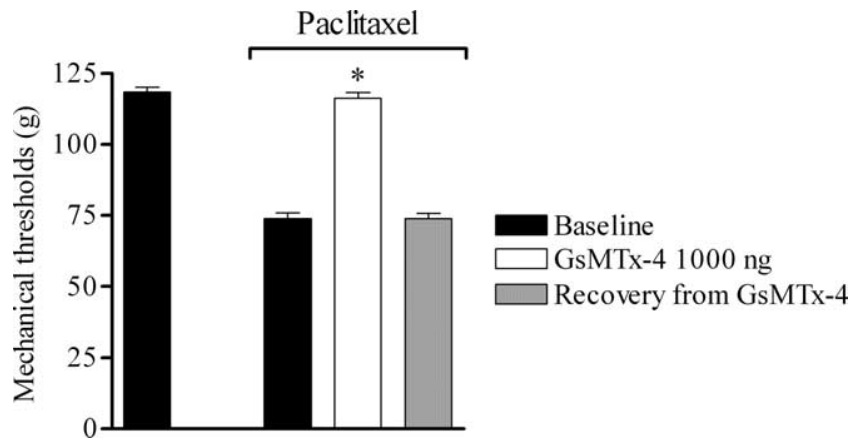

Figure 4. GsMTx-4 reverses paclitaxel chemotherapy-induced mechanical hyperalgesia. Rats treated with paclitaxel (daily for 10 d) develop mechanical hyperalgesia (118 $\pm 2 \mathrm{~g}, n=$ 12 before and $74 \pm 3 \mathrm{~g}, n=6$ after paclitaxel; $p<0.001$, Tukey's posthoc multiple comparison test). Intradermal injection of GsMTX-4 in the hindpaw 30 min before mechanical nociceptive threshold testing reversed the mechanical hyperalgesia ( $74 \pm 3 \mathrm{~g}$ before GsMTx-4 and $116 \pm$ $2 \mathrm{~g}$ after; $n=6 ; p<0.001$, Tukey's post hoc multiple comparison test). Twenty-four hours after the administration of GsMTX-4, mechanical nociceptive thresholds were not significantly different from pre-GsMTx-4 paw-withdrawal thresholds ( $n=6$ for each group; $p>0.05$, Tukey's post hoc multiple comparison test).

for $5 \mathrm{~min}$ and challenged with a 30\% hypotonic solution containing $\mathrm{PGE}_{2}, 5-\mathrm{HT}$, and GsMTx-4 for 3 min. GsMTx-4 caused a decrease in the hypotonicity-induced increase in $\left[\mathrm{Ca}^{2+}\right]_{\mathrm{i}}$ in both TRPV4 genotypes (Fig. $5 B)(p<0.05$, paired Student's $t$ test).

\section{TRPC1 and TRPC6 also contribute to hyperalgesia for} mechanical and hypotonic stimuli

GsMTx-4 inhibits TRPC1 and TRPC6 (Spassova et al., 2006; Bowman et al., 2007), two members of the canonical subfamily of TRP channels. TRPC1 and TRPC6 channels respond to mechanical and hypotonic stimuli in non-neuronal cells (Maroto et al., 2005; Spassova et al., 2006) and are present in DRG neurons (Elg et al., 2007; Kress et al., 2008). Thus, we investigated whether TRPV4, TRPC1, and TRPC6 are coexpressed in DRG neurons. We harvested the cytoplasm of 47 DRG neurons and ran multiplex single-cell RT-PCR. Of the 47 neurons tested, 14 did not express any of the three candidates. Of the remaining 33 neurons, 16 coexpressed TRPV4, TRPC1, and TRPC6, whereas the other 17 expressed either one or two (Fig. 5C). Because level of mRNAs do not reflect the level of protein in the plasma membrane, we tested whether we could detect TRPV4, TRPC1, and TRPC6 in membrane fractions isolated from cultured lumbar DRG neurons by Western blot analysis. On day 2 in culture, neurons were homogenized and membrane fractions were isolated by sucrose gradient centrifugation. Proteins were then separated on an electrophoresis gel and analyzed by Western blotting. As shown in Figure $5 D$, we detected the expected bands at 120 and $110 \mathrm{kDa}$ for TRPC1 and TRPC6, respectively, as well as the expected doublet bands at 98 and $107 \mathrm{kDa}$ for TRPV4. This result was reproducible in three independent experiments.

Next, to investigate the contribution of TRPC1 and TRPC6 channels to mechanotransduction, rats received spinal intrathecal administrations of antisense or mismatch ODN for TRPC1 or

C-fibers that were soup sensitive $(n=15)$ vs soup insensitive $(n=11)$ or between C-fibers that were GsMTX-4 sensitive or GsMTX-4 insensitive ( $p>0.05$, Tukey's post hoc multiple comparison test). $\boldsymbol{D}$, Response of sensitized C-fibers to suprathreshold (10 g) mechanical stimulation before $(\boldsymbol{\bullet})$ and after $(\boldsymbol{\Lambda})$ intradermal injection of $\mathrm{PGE}_{2}$ and 5 -HT and after a subsequent intra-

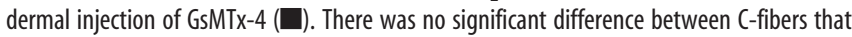
were GSMTx-4-sensitive or GsMTX-4-insensitive. 
A

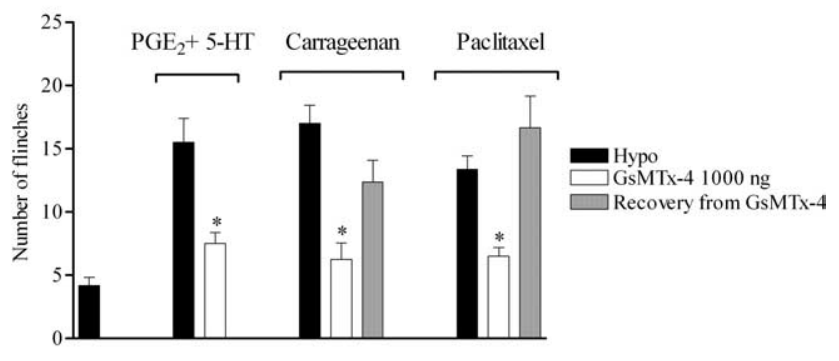

B

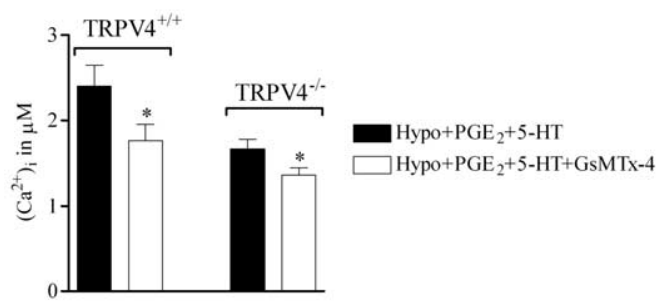

C

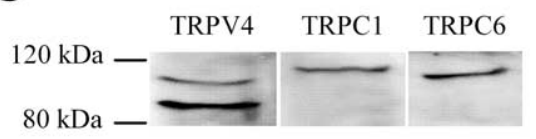

D

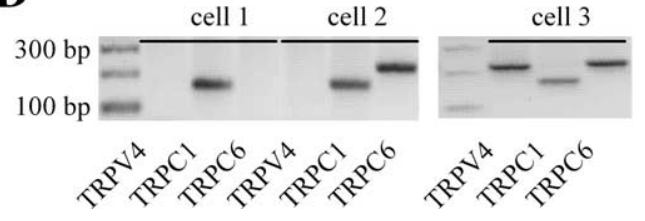

Figure 5. GsMTx-4 also markedly reduces nociceptive flinching in response to hypotonic stimulation. $A$, Rats treated with $\mathrm{PGE}_{2}$ and 5 -HT, carrageenan, or paclitaxel have, respectively, a 4-, 3.6-, and 3.2-fold increase in the number of nociceptive flinches in response to an intradermal injection of hypotonic solution (Hypo) (10 $\mu$ l of deionized water, $17 \mathrm{~m} 0 \mathrm{sm}$ ) compared with control rats. GSMTX-4 markedly reduced the number of flinches in $\mathrm{PGE}_{2}$ and 5 - $\mathrm{HT}$-treated (16 \pm $2, n=12$ before and $8 \pm 1, n=6$ after GsMTx-4), carrageenan-treated (17 $\pm 1, n=12$ before and $6 \pm 1, n=8$ after GSMTx-4), and paclitaxel-treated ( $13 \pm 2, n=10$ before and $7 \pm 1, n=6$ after GsMTx-4) rats. All asterisks, $p<0.05$, Tukey's post hoc multiple comparison test. Twenty-four hours after the administration of GSMTx-4, its effect was no longer present, and there was no significant difference in the number of flinches between the different groups of rats ( $p>0.05$, Tukey's multiple comparison test). $\boldsymbol{B}$, Small-diameter ( $\leq 25 \mu \mathrm{m}$ ) DRG neurons from $\mathrm{TRPV}^{+/+}$and $\mathrm{TRPV}^{-/-}$mice were first challenged with a $30 \%$ hypotonic solution containing PGE $\mathrm{P}_{2}$ and 5-HT (10 $\mu \mathrm{m}$ each) for $3 \mathrm{~min}$ and then challenged with a 30\% hypotonic solution containing PGE, $5-H T$, and GsMTx-4 $(500 \mathrm{~nm})$ for 3 min. The increase in $\left[\mathrm{Ca}^{2+}\right]_{\mathrm{i}}$ induced by the hypotonic solution containing $\mathrm{PGE}_{2}$ and $5-\mathrm{HT}$ is reduced in the presence of GSMTX- 4 in TRPV4 $4^{+/+}$mice $(2.4 \pm 0.2 \mu \mathrm{m}$ before and $1.7 \pm 0.2 \mu \mathrm{m}$ after GsMTx-4; $n=24$; $p=0.007$, paired Student's $t$ test $)$ as well as in TRPV $4^{-/-}$mice $(1.7 \pm 0.1 \mu \mathrm{m}$ before and $1.4 \pm 0.1 \mu \mathrm{m}$ after GsMTx-4; $n=14 ; p=0.03$, paired Student's $t$ test). C, Dissociated lumbar DRG neurons were cultured for $2 \mathrm{~d}$. Cells were scraped in homogenization buffer, and subcellular fractionation was performed. The membrane fraction was separated on an electrophoresis gel and transferred to a polyvinylidene difluoride membrane. The membrane was then probed in parallel with anti-TRPV4 (1:500), anti-TRPC1 (1:500), and anti-TRPC6 (1:500) antibodies. The expected bands at $120 \mathrm{kDa}$ for TRPC1, $110 \mathrm{kDa}$ for TRPC6, and the doublet bands at 98 and 107 $\mathrm{kDa}$ for TRPV4 were detected. $\boldsymbol{D}$, The cytoplasm of 47 DRG neurons was harvested after $2 \mathrm{~d}$ in culture, and multiplex single-cell RT-PCR was performed. Neurons expressed from none to all three of the mRNAs of interest; here we show the example of neurons expressing mRNA for TRPC1 (168 bp) or TRPC1 and TRPC6 (228 bp) or TRPC1, TRPC6, and TRPV4 (221bp).

TRPC6, daily for $3 \mathrm{~d}$. As shown in Figure 6A, TRPC1 or TRPC6 antisense reversed the decrease in mechanical threshold induced by $\mathrm{PGE}_{2}$ and 5 -HT compared with mismatch ODN-treated rats. The effect of the antisense was reversible; $4 \mathrm{~d}$ after the last ODN injection, the change in mechanical paw-withdrawal threshold induced by $\mathrm{PGE}_{2}$ and 5-HT was not significantly different from

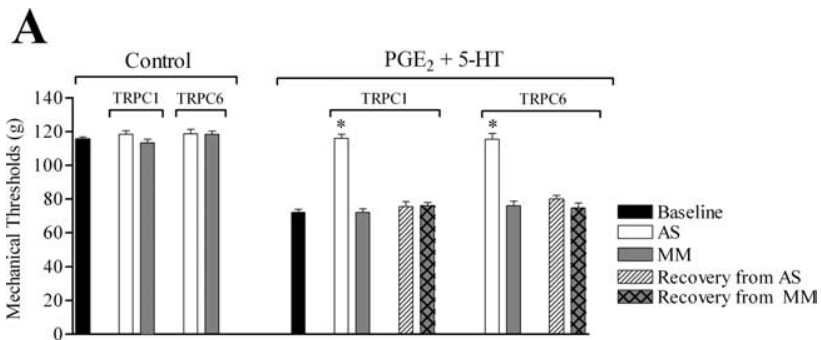

B

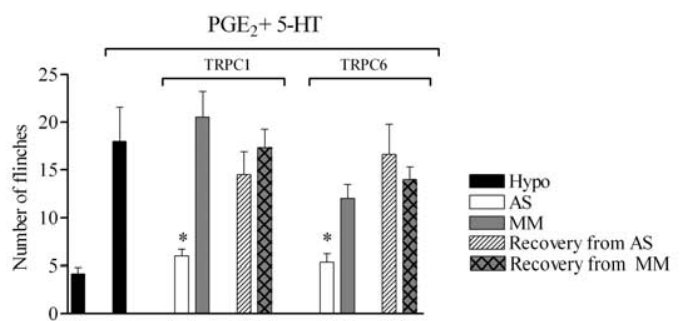

C

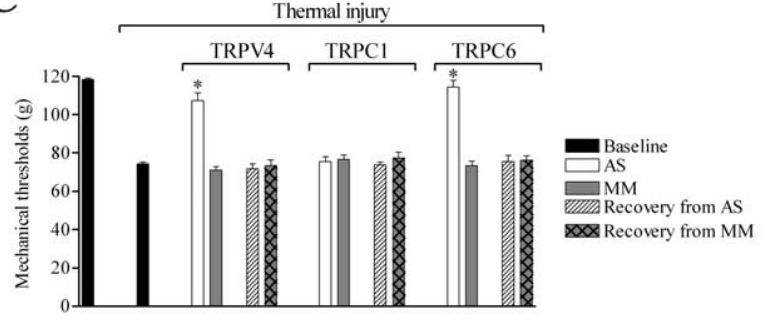

D

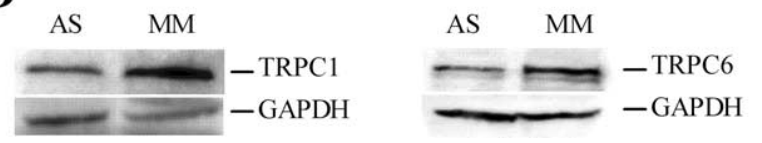

Figure 6. Hyperalgesia to mechanical and hypotonic stimuli induced by $\mathrm{PGE}_{2}$ and $5-\mathrm{HT}$ is TRPC 1 and TRPC 6 dependent. $A$, Treatment with TRPC 1 or TRPC 6 antisense (AS) ODN for $3 \mathrm{~d}$ did not affect baseline nociceptive mechanical threshold in control rats $(n=24$ for baseline and $n=6$ for antisense and mismatch-treated rats; $p>0.05$, Tukey's post hoc multiple comparison test). In contrast, the mechanical hyperalgesia induced by $\mathrm{PGE}_{2}$ and 5 -HT was reversed by treatment with TRPC 1 or TRPC 6 antisense compared with mismatch-treated (MM) rats ( $n=18$ for baseline and $n=6$ for antisense and mismatch-treated rats; ${ }^{*} p<0.001$, Tukey's post hoc multiple comparison test). The effect of the antisense was reversible; $4 \mathrm{~d}$ after the last ODN injection, the mechanical hyperalgesia induced by $\mathrm{PGE}_{2}$ and 5 - $\mathrm{HT}$ was not significantly different from pre-ODN baseline ( $p>0.05$, Tukey's multiple-comparison test). $\boldsymbol{B}$, Treatment with TRPC1 or TRPC 6 antisense also markedly reduced the number of flinches in response to intradermal injection of hypotonic solution (Hypo) in the presence of $\mathrm{PGE}_{2}$ and $5-\mathrm{HT}$ compared with mismatch-treated rats $(n=6$ for hypotonic solution in presence of PGE 2 plus $5-\mathrm{HT}$ and $n=6$ for each ODN group; ${ }^{*} p<0.001$, Tukey's post hoc multiple comparison test). C, Paw-withdrawal thresholds were markedly decreased $24 \mathrm{~h}$ after thermal injury $(118 \pm 1 \mathrm{~g}$ before vs $74 \pm 1 \mathrm{~g}$ after thermal injury; $n=36 ; p<0.0001$, paired Student's $t$ test). Treatment with TRPV4 or TRPC6 antisense for $3 \mathrm{~d}$ markedly reversed the mechanical hyperalgesia compared with mismatch $0 D N$-treated rats ( $n=6$ for all groups). Again, the effect of the antisense treatment was reversible; there was no significant difference in the mechanical thresholds between baseline and ODN-treated groups $4 \mathrm{~d}$ after the last ODN injection ( $n=36$ for baseline and $n=6$ for each ODN group; all $p>0.05$, Tukey's post hoc multiple comparison test). In contrast, TRPC1 antisense did not affect the mechanical hyperalgesia induced by thermal injury. $\mathbf{D}$, There was a $37 \pm 4$ and $32 \pm 7 \%$ decrease in TRPC 1 and TRPC 6 protein expression level, respectively $(n=$ 3 for all groups; $p<0.05$, unpaired Student's $t$ test) in DRG of rats treated with antisense (AS) ODN compared with mismatch ODN-treated rats (MM). The amount of protein loaded in each lane was normalized by probing the membrane with an anti-GAPDH antibody.

the pre-ODN response (Fig. $6 A)(p>0.05$, Tukey's post hoc multiple comparison test; $n=6$ for each ODN group and $n=18$ for baseline). TRPC 1 and TRPC6 antisense did not affect baseline nociceptive mechanical threshold, and there was no significant 
difference between the groups of rats treated with ODNs and untreated control rats (Fig. $6 A)(n=6$ for each ODN group and $n=24$ for baseline; $p>0.05$, Tukey's post hoc multiple comparison test).

To further test for a role of TRPC1 and TRPC6 in the hyperalgesia induced by inflammatory mediators, we evaluated whether TRPC1 and TRPC6 antisense treatment reduces the number of flinches in response to hypotonicity. As shown in Figure $6 B$, treatment with TRPC1 and TRPC6 antisense reduced the number of flinches in inflammatory soup-treated rats by 71 and 56\%, respectively, compared with mismatch treatment $(n=$ 6 for all groups; $p<0.05$, Tukey's post hoc multiple comparison test). Again, the effect of TRPC1 or TRPC6 antisense treatment was reversible; $4 \mathrm{~d}$ after the last injection of ODN, the number of flinches in response to hypotonicity was similar to the pre-ODN baseline ( $n=6$ for all groups; $p>0.05$, Tukey's post hoc multiple comparison test).

Treatment with TRPC1 or TRPC6 antisense markedly reduces the enhanced nociceptive behaviors to mechanical and hypotonic stimuli in the presence of inflammatory soup, to the same extent as treatment with TRPV4 antisense (Alessandri-Haber et al., 2006). Moreover, like TRPV4, although TRPC1 and TRPC6 make no apparent contribution to baseline mechanical thresholds, they participate in mechanical hyperalgesia. These results suggest that TRPC1 and TRPC6 channels function with TRPV4 to produce mechanical hyperalgesia.

To further investigate a possible functional interaction among these three TRP channels, we tested the effect of antisense to TRPV4, TRPC1, and TRPC6 on a model of inflammation with a very different etiology, thermal burn injury. We recently demonstrated that a partial-thickness burn of less than $1 \%$ total body surface area on the dorsum of rat hindpaw induces robust mechanical hyperalgesia that lasts up to $25 \mathrm{~d}$ (Summer et al., 2006, 2007, 2008). Mechanical nociceptive thresholds were significantly decreased $24 \mathrm{~h}$ after the thermal injury (Fig. 6C) (118 $\pm 1 \mathrm{~g}$ before vs $74 \pm 1 \mathrm{~g}$ after thermal injury; $n=36$; $p<0.0001$, paired Student's $t$ test). As shown in Figure $6 C$, treatment with TRPV4 or TRPC6 antisense ODNs reversed the mechanical hyperalgesia compared with mismatch-treated rats ( $n=6$ for all groups; $p<0.001$, Tukey's post hoc multiple comparisons test). In contrast, antisense ODNs to TRPC1 did not affect the thermal injury-induced mechanical hyperalgesia ( $n=36$ for baseline and $n=6$ for both antisense and mismatch treated; $p>0.05$, Tukey's post hoc multiple comparison test). These results suggest that, although TRPC1, TRPC6, and TRPV4 "cooperate" in the mechanism of mechanical hyperalgesia induced by intradermal injection of inflammatory mediators, the functional coupling between these three channels may depend on the etiology of the hyperalgesia.

To confirm that the reduction in the nociceptive behaviors induced by TRPC1 or TRPC6 antisense administration is associated with a decrease in the level of TRPC1 and TRPC6 protein, respectively, we performed Western blot analyses. Rats were treated with antisense or mismatch ODN for $3 \mathrm{~d}$, and, on day 4, L5-L6 DRGs were harvested and processed for Western blot analysis. As shown in Figure 6D, TRPC1 and TRPC6 antisense treatment caused a $37 \pm 4$ and $32 \pm 7 \%$ decrease, respectively, in the level of expression of TRPC1 and TRPC6 protein compared with mismatch-treated rats $(n=3$ for each group; $p<0.05$, unpaired Student's $t$ test).

\section{Treatment with TRPC6, but not TRPC1, antisense reverses $4 \alpha$-PDD-induced hyperalgesia}

The phorbol ester $4 \alpha$-PDD, an inactive analog of phorbol 12myristate 13 -acetate, activates TRPV4 by direct binding to a transmembrane region (Watanabe et al., 2002; Vriens et al., 2007). Intradermal injection of $4 \alpha$-PDD $(1 \mu \mathrm{g} / 2.5 \mu \mathrm{l})$ in the hindpaw, $30 \mathrm{~min}$ before mechanical testing, induced mechanical hyperalgesia (Fig. $7 A)(120 \pm 1 \mathrm{~g}, n=8$ for baseline vs $76 \pm 1 \mathrm{~g}$, $n=8$ after $4 \alpha$-PDD; $p<0.0001$, paired Student's $t$ test). To determine whether TRPC1 and TRPC6 are necessary for TRPV4 function, we investigated whether treatment with TRPC1 or TRPC6 antisense could inhibit the mechanical hyperalgesia induced by $4 \alpha$-PDD. As shown in Figure $7 B$, TRPC6 antisense reversed $4 \alpha$-PDD-induced mechanical hyperalgesia $(76 \pm 1 \mathrm{~g}$, $n=8$ for $4 \alpha$-PDD baseline; $123 \pm 5 \mathrm{~g}, n=6$ for antisense and $71 \pm 2 \mathrm{~g}, n=6$ for mismatch-treated rats; $p<0.001$, Tukey's post hoc multiple comparison test). In contrast, TRPC1 antisense did not significantly affect the decrease in nociceptive mechanical threshold induced by $4 \alpha$-PDD (Fig. $7 B$ ) ( $p>0.05$, Tukey's post hoc multiple comparison test). Four days after the last ODN injection, the decrease in nociceptive mechanical threshold induced by $4 \alpha$-PDD was not significantly different among TRPC6 ODN pretreated rats and pre-ODN baseline $(p>0.05$, Tukey's post hoc multiple comparison test).

Next, we tested whether intradermal injection of $4 \alpha$-PDD in rat hindpaw induced nociceptive flinching. As shown in Figure $7 C, 4 \alpha$-PDD induces flinches $(15 \pm 1 ; n=6)$. Moreover, $4 \alpha-$ PDD sensitizes nociceptors to the hypotonic stimulus; rats pretreated with $4 \alpha$-PDD have a sixfold increase in the number of nociceptive flinches in response to an injection of hypotonic solution (Fig. 7C) $(4 \pm 1, n=12$ for hypotonic in the absence and $24 \pm 3, n=10$ in the presence of $4 \alpha$-PDD; $p<0.0001$, unpaired Student's $t$ test). Treatment with TRPC6 antisense reduced the number of flinches induced by hypotonic solution in $4 \alpha$-PDDtreated paws by $60 \%$ compared with mismatch (Fig. 7C) $(24 \pm 3$, $n=10$ for hypotonic in the presence of $4 \alpha$-PDD, $8 \pm 1, n=6$ for antisense-treated rats and $20 \pm 1, n=6$ for mismatch-treated rats; $p<0.001$, Tukey's post hoc multiple comparison test). Again, the effect of TRPC6 antisense was reversible; $4 \mathrm{~d}$ after the last ODN injection, there was no significant difference between the two groups of rats ( $p>0.05$, Tukey's post hoc multiple comparison test). In contrast, there was no significant difference in the number of flinches in response to intradermal injection of hypotonic solution in presence of $4 \alpha$-PDD between rats treated with TRPC1 antisense and TRPC1 mismatch (Fig. 7C) $(n=10$ for $4 \alpha$-PDD plus hypotonic and $n=6$ for ODN groups; $p>0.05$, Tukey's post hoc multiple comparison test). These results suggest that TRPC6 closely interacts with TRPV4 in primary afferent nociceptor sensitization.

\section{Discussion}

We demonstrated recently that TRPV4 plays a fundamental role in mechanical hyperalgesia induced by diverse inflammatory and neuropathic agents that produce pain by various mechanisms of action (Alessandri-Haber et al., 2006, 2008). However, SACs contribute to the detection of mechanical stimuli in DRG neurons and can also be sensitized by hyperalgesic agents such as $\mathrm{PGE}_{2}$ (McCarter et al., 1999; Cho et al., 2002; $\mathrm{Hu}$ and Lewin, 2006). Therefore, we investigated whether SACs may interact with TRPV4 to produce mechanical hyperalgesia by testing the selective SAC blocker GsMTx-4 on mechanical hyperalgesia associated with different models of inflammatory or neuropathic pain in the rat. 


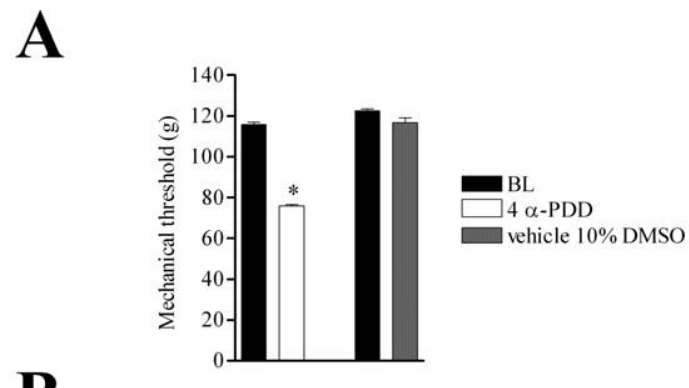

B
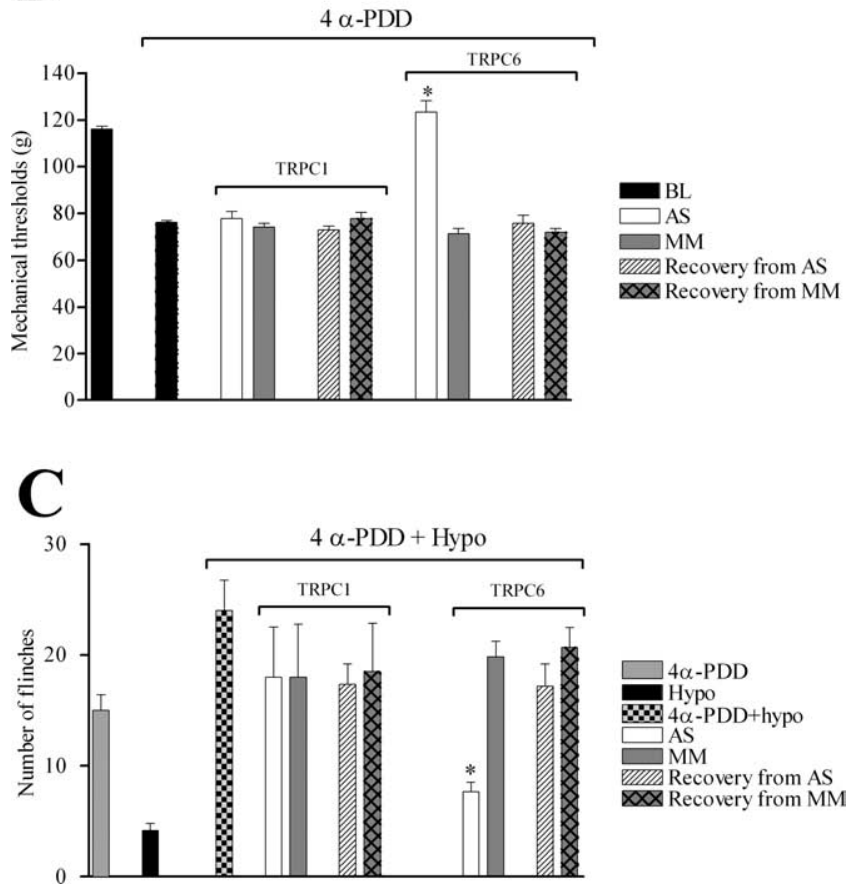

Figure 7. Antisense oligodeoxynucleotides to TRPC6 reverses $4 \alpha$-PDD-induced hyperalgesia to mechanical and hypotonic stimuli. $A$, Intradermal injection of $4 \alpha$-PDD $(1 \mu \mathrm{g} / 2.5 \mu l)$ in rat hindpaw $30 \mathrm{~min}$ before measurement of nociceptive thresholds induced a significant decrease in mechanical thresholds $\left(120 \pm 1 \mathrm{~g}, n=8\right.$ for baseline vs $76 \pm 1 \mathrm{~g}, ; n=8$ after $4 \alpha$-PDD; ${ }^{*} p<0.0001$, paired Student's $t$ test). In contrast, intradermal injection of the vehicle, $10 \%$ DMSO, had no significant effect on mechanical thresholds ( $n=4$ before and after 10\% DMSO; $p>0.05$, paired Student's $t$ test). BL, Baseline. $\boldsymbol{B}$, Treatment with TRPC6 antisense (AS) ODN for $3 \mathrm{~d}$ reversed the mechanical hyperalgesia induced by $4 \alpha$-PDD compared with mismatch-treated (MM) rats ( $n=8$ for baseline and $n=6$ for each ODN group; ${ }^{*} p<0.001$, Tukey's post hoc multiple comparison test). The effect of the antisense was reversible; $4 \mathrm{~d}$ after the last $0 \mathrm{DN}$ injection, the mechanical thresholds was similar in baseline and ODN-treated rats ( $p>0.05$, Tukey's multiple comparison test). In contrast, treatment with TRPC1 antisense 0DN does not affect the decrease in mechanical threshold induced by $4 \alpha-\operatorname{PDD}$ ( $p>0.05$, Tukey's posthocmultiple comparison test). $C$, Intradermal injection of $4 \alpha$-PDD in rathindpaw induces a significant number of flinches $(15 \pm 1, n=6)$. Moreover, rats pretreated with $4 \alpha$-PDD have a sixfold increase in the number of nociceptive flinches in response to an injection of hypotonic solution ( $4 \pm 1, n=12$ for hypotonic in the absence and $24 \pm 3, n=10$ in the presence of $4 \alpha$-PDD; $p<$ 0.0001 , unpaired Student's $t$ test). Treatment with TRPC 6 antisense ODN markedly reduced the number of flinches induced by intradermal injection of hypotonic solution in the presence of $4 \alpha$-PDD compared with mismatch-treated rats ( $24 \pm 3, n=10$ for baseline $4 \alpha$-PDD plus hypotonic solution; $8 \pm 1, n=6$ for antisense-treated rats; and $20 \pm 1, n=6$ for mismatch-treated rats; ${ }^{*} p<0.001$, Tukey's post hoc multiple comparison test). Again the effect of antisense was reversible; $4 \mathrm{~d}$ after the last ODN injection, the number of flinches was not significantly different between TRPC 6 antisenseand mismatch-treated rats. In contrast, antisense ODN to TRPC1 did not affect the number of flinches ( $n=10$ for baseline $4 \alpha$-PDD plus hypotonic solution and $n=6$ for $0 D N$ groups; $p>0.05$, Tukey's post hoc multiple comparison test).

We demonstrate that, although intradermal injection of GsMTx-4 does not affect baseline nociceptive mechanical threshold in behavioral studies nor C-fiber threshold and responses to mechanical stimuli, it reverses mechanical hyperalgesia and markedly reduces the response of sensitized C-fiber primary afferents to mechanical stimuli. Park et al. (2008) reported an effect of GsMTx-4 on baseline nociceptive mechanical thresholds, but they used a systemic intraperitoneal injection, whereas we used a peripheral intradermal injection at the site of nociceptive testing, which restricts the action of GsMTx-4 to the peripheral terminal of the nociceptor. Moreover, GsMTx-4 inhibits the hyperalgesia for mechanical and hypotonic stimuli, induced by very different hyperalgesic agents ( $\mathrm{PGE}_{2}$ and $5-\mathrm{HT}$, carrageenan and paclitaxel), and does it to the same extent as treatment with TRPV4 antisense ODN (Alessandri-Haber et al., 2004, 2006, 2008).

GsMTx-4 has been shown to inhibit TRPC1 and TRPC6 channels (Spassova et al., 2006; Bowman et al., 2007); both channels are strongly expressed in isolectin B4-positive DRG neurons (Kress et al., 2008) and respond to both hypotonic and mechanical stimuli (Maroto et al., 2005; Spassova et al., 2006). We demonstrate that antisense ODN for TRPC1 or TRPC6 reverses the hyperalgesia to hypotonic and mechanical stimuli induced by peripheral administration of $\mathrm{PGE}_{2}$ and $5-\mathrm{HT}$ to the same extent as TRPV4 antisense (Alessandri-Haber et al., 2006). Of note, we demonstrated recently that intradermal injection of $\mathrm{PGE}_{2}$ and 5-HT does not induce hyperalgesia to hypotonic and mechanical stimuli in mice lacking a functional TRPV4 gene or in rats treated with TRPV4 antisense ODN (Alessandri-Haber et al., 2005, 2006). In addition, like TRPV4, TRPC1 and TRPC6 make no apparent contribution to baseline mechanical nociceptive threshold. Finally, TRPV4, TRPC1, and TRPC6 protein are detectable in membrane fractions of cultured DRG neurons, and, whereas $\sim 70 \%$ of cultured small-diameter neurons expressed at least one of the three TRPs, approximately half of these neurons (49\%, 16 neurons of 33 tested) coexpress all three. Our findings suggest that these channels could functionally interact within sensitized sensory neurons to manifest mechanical hyperalgesia. Of note, these three channels are also expressed and functional in keratinocytes (Chung et al., 2003, 2004; Cai et al., 2005, 2006) in which they could interact to sensitize nociceptors indirectly. However, in the present study, antisense to TRPV4, TRPC1, and TRPC6 were administered by spinal intrathecal injection; thus, among the cells present in the skin, only sensory neurons are exposed to the ODN.

Our results do not discriminate the nature of the interaction between TRPV4, TRPC1, and TRPC6, but they do suggest that, although these channels cooperate in the development of mechanical hyperalgesia, they may have distinctive roles. TRPC6 and TRPV4 contribute to mechanical hyperalgesia of diverse etiology (i.e., $\mathrm{PGE}_{2}$ and 5-HT, thermal burn, and $4 \alpha$-PDD), whereas TRPC1 only contributes to that induced by $\mathrm{PGE}_{2}$ and 5-HT. The finding that treatment with TRPC6 antisense reverses the $4 \alpha$-PDD-induced hyperalgesia to hypotonic and mechanical stimuli suggests that TRPC6 closely interacts with TRPV4 because $4 \alpha$-PDD binds directly to transmembrane regions 3 and 4 of the TRPV4 channel (Vriens et al., 2007). Importantly, the interaction between TRPV4 and TRPC6 in sensitized nociceptors is specific to mechanical transduction because intraplantar injection of $4 \alpha$-PDD induces mechanical hyperalgesia (Grant et al., 2007) but not thermal hyperalgesia (Souza et al., 2002). Interestingly, TRPV4 was shown recently to couple with another TRP channel, TRPP2, to form a polymodal sensory channel complex in cilia (Köttgen et al., 2008). We suggest that TRPC6 may be similarly coupled to TRPV4, as a component of a molecular mechanotransduction complex comprising, but not limited to, TRPV4 channels, integrins, and Src tyrosine kinases (AlessandriHaber et al., 2008); of note, in this regard, members of the Src 
family kinase can also directly bind to TRPC6 and regulate its activity (Hisatsune et al., 2004), and TRPC6 has been shown to participate in mechanotransduction in non-neuronal cells (Dietrich and Gudermann, 2007; Dietrich et al., 2007a). Also, both TRPV4 and TRPC6 have been shown to interact with large-conductance calcium-dependent potassium channels in non-neuronal cells (Fernández-Fernández et al., 2008; Kim et al., 2009). Whether TRPC6 can be directly activated by stretch remains controversial (Maroto et al., 2005; Spassova et al., 2006; Dietrich et al., 2007b; Gottlieb et al., 2008; Sharif-Naeini et al., 2008), and it was suggested recently that, in vascular smooth muscle cells, TRPC6 may not be primarily gated by membrane stretch but rather by an agonistindependent activation of $\mathrm{G}_{\mathrm{q} / 11}$-coupled receptors that in turn activates protein $\mathrm{G}$ and phospholipase C (PLC) pathways (Mederos et al., 2008). A similar type of activation in nociceptors may be one mechanism by which TRPC6 contributes to mechanical hyperalgesia but not to baseline nociceptive mechanical thresholds. We also cannot rule out the existence of TRPV4 and TRPC6 heteromers. Additional investigation is needed to understand the role of TRPC6 in the transduction of mechanical stimuli in sensitized nociceptors.

TRPC channels can function as store-operated channels (SOCs) or receptor-operated channels (ROCs) through PLC activation, and, whereas TRPC6 has been shown to be activated by diacylglycerol but not by internal calcium store depletion, several studies describe TRPC1 as a strong candidate for an SOC in neuronal cells (Ambudkar and Ong, 2007; Rychkov and Barritt, 2007). TRPC1 has been shown to colocalize with calcium signaling proteins such as PLC, protein $\mathrm{G} \alpha_{\mathrm{q} / 11}$, inositol triphosphate receptors $\left(\mathrm{IP}_{3} \mathrm{Rs}\right)$, and $\mathrm{B}_{2}$ bradykinin receptor (Lockwich et al., 2000; Delmas et al., 2002; Yuan et al., 2003). Moreover, inflammatory mediators act on G-protein-coupled receptors (GPCRs), and TRPC channels can directly interact with GPCRs (Ambudkar and Ong, 2007). Thus, TRPC1 might contribute in inflammatory soup-induced mechanical hyperalgesia as an SOC or a necessary accessory protein required for the activation of a SOC; the binding of $\mathrm{PGE}_{2}$ and 5-HT to their respective G-protein-coupled receptors could initiate the activation of phospholipase $\mathrm{C}$, the generation of $\mathrm{IP}_{3}$, and calcium release by $\mathrm{IP}_{3} \mathrm{Rs}$, which in turn activate TRPC1 (Fig. 8). The $\mathrm{IP}_{3} \mathrm{R}$ has been shown to directly interact with TRPC1 (Rychkov and Barritt, 2007) as well as TRPV4 (Garcia-Elias et al., 2008). How TRPC1 interacts with TRPV4 and TRPC6 remains to be elucidated. However, the existence of heteromers and interactions with other molecules, such as cytoskeletal proteins, are likely to also participate in the mechanism of mechanical transduction. The presence of TRPC channels, some acting as SOCs others as ROCs, within the same cell (Bréchard et al., 2008), and their interaction with other TRP family members reflects not only the complexity of the TRP family of channels but also its physiological importance in nociceptor function.

In conclusion, we demonstrate for the first time that, although TRPC1 and TRPC6 channels do not contribute to baseline me- chanical nociceptive threshold, they participate in the detection of mechanical stimuli in sensitized primary afferent nociceptors. We suggest that, although TRPV4, TRPC6, and TRPC1 have distinctive roles, they cooperate in a mechanism mediating mechanical hyperalgesia and primary afferent nociceptor sensitization. Moreover, TRPC6 emerges like an attractive candidate as a component of signaling complexes, including TRPV4, integrins, and Src tyrosine kinases, whose function is to transduce mechanical stimuli in the setting of inflammation or nerve injury.

\section{References}

Alessandri-Haber N, Yeh JJ, Boyd AE, Parada CA, Chen X, Reichling DB, Levine JD (2003) Hypotonicity induces TRPV4-mediated nociception in rat. Neuron 39:497-511.

Alessandri-Haber N, Dina OA, Yeh JJ, Parada CA, Reichling DB, Levine JD (2004) Transient receptor potential vanilloid 4 is essential in chemotherapy-induced neuropathic pain in the rat. J Neurosci 24:4444-4452.

Alessandri-Haber N, Joseph E, Dina OA, Liedtke W, Levine JD (2005) TRPV4 mediates pain-related behavior induced by mild hypertonic stimuli in the presence of inflammatory mediator. Pain 118:70-79.

Alessandri-Haber N, Dina OA, Joseph EK, Reichling D, Levine JD (2006) A transient receptor potential vanilloid 4-dependent mechanism of hyperalgesia is engaged by concerted action of inflammatory mediators. J Neurosci 26:3864-3874.

Alessandri-Haber N, Dina OA, Joseph EK, Reichling DB, Levine JD (2008) Interaction of transient receptor potential vanilloid 4, integrin, and SRC tyrosine kinase in mechanical hyperalgesia. J Neurosci 28:1046-1057.

Aley KO, Levine JD (2001) Rapid onset pain induced by intravenous streptozotocin in the rat. J Pain 2:146-150.

Aley KO, Martin A, McMahon T, Mok J, Levine JD, Messing RO (2001) Nociceptor sensitization by extracellular signal-regulated kinases. J Neurosci 21:6933-6939.

Ambudkar IS, Ong HL (2007) Organization and function of TRPC channelosomes. Pflugers Arch 455:187-200.

Bowman CL, Gottlieb PA, Suchyna TM, Murphy YK, Sachs F (2007) Mechanosensitive ion channels and the peptide inhibitor GsMTx-4: history, properties, mechanisms and pharmacology. Toxicon 49:249-270.

Bréchard S, Melchior C, Plançon S, Schenten V, Tschirhart EJ (2008) Store- 
operated $\mathrm{Ca}(2+)$ channels formed by TRPC1, TRPC6 and Orail and non-store-operated channels formed by TRPC 3 are involved in the regulation of NADPH oxidase in HL-60 granulocytes. Cell Calcium 44:492-506.

Cai S, Fatherazi S, Presland RB, Belton CM, Izutsu KT (2005) TRPC channel expression during calcium-induced differentiation of human gingival keratinocytes. J Dermatol Sci 40:21-28.

Cai S, Fatherazi S, Presland RB, Belton CM, Roberts FA, Goodwin PC, Schubert MM, Izutsu KT (2006) Evidence that TRPC1 contributes to calcium-induced differentiation of human keratinocytes. Pflugers Arch 452:43-52.

Chen X, Levine JD (1999) NOS inhibitor antagonism of PGE2-induced mechanical sensitization of cutaneous C-fiber nociceptors in the rat. J Neurophysiol 81:963-966.

Cho H, Shin J, Shin CY, Lee SY, Oh U (2002) Mechanosensitive ion channels in cultured sensory neurons of neonatal rats. J Neurosci 22:1238-1247.

Chung MK, Lee H, Caterina MJ (2003) Warm temperatures activate TRPV4 in mouse 308 keratinocytes. J Biol Chem 278:32037-32046.

Chung MK, Lee H, Mizuno A, Suzuki M, Caterina MJ (2004) TRPV3 and TRPV4 mediate warmth-evoked currents in primary mouse keratinocytes. J Biol Chem 279:21569-21575.

Dalrymple A, Slater DM, Poston L, Tribe RM (2004) Physiological induction of transient receptor potential canonical proteins, calcium entry channels, in human myometrium: influence of pregnancy, labor, and interleukin-1 beta. J Clin Endocrinol Metab 89:1291-1300.

Delmas P, Wanaverbecq N, Abogadie FC, Mistry M, Brown DA (2002) Signaling microdomains define the specificity of receptor-mediated $\operatorname{Ins} \mathrm{P}(3)$ pathways in neurons. Neuron 34:209-220.

Dietrich A, Gudermann T (2007) Trpc6. Handb Exp Pharmacol 2007:125-141.

Dietrich A, Kalwa H, Fuchs B, Grimminger F, Weissmann N, Gudermann T (2007a) In vivo TRPC functions in the cardiopulmonary vasculature. Cell Calcium 42:233-244.

Dietrich A, Kalwa H, Storch U, Mederos y Schnitzler M, Salanova B, Pinkenburg O, Dubrovska G, Essin K, Gollasch M, Birnbaumer L, Gudermann T (2007b) Pressure-induced and store-operated cation influx in vascular smooth muscle cells is independent of TRPC1. Pflugers Arch 455:465-477.

Dina OA, Chen X, Reichling D, Levine JD (2001) Role of protein kinase Cepsilon and protein kinase A in a model of paclitaxel-induced painful peripheral neuropathy in the rat. Neuroscience 108:507-515.

Drew LJ, Rugiero F, Cesare P, Gale JE, Abrahamsen B, Bowden S, Heinzmann S, Robinson M, Brust A, Colless B, Lewis RJ, Wood JN (2007) Highthreshold mechanosensitive ion channels blocked by a novel conopeptide mediate pressure-evoked pain. PLoS ONE 2:e515.

Elg S, Marmigere F, Mattsson JP, Ernfors P (2007) Cellular subtype distribution and developmental regulation of TRPC channel members in the mouse dorsal root ganglion. J Comp Neurol 503:35-46.

Fernández-Fernández JM, Andrade YN, Arniges M, Fernandes J, Plata C, Rubio-Moscardo F, Vázquez E, Valverde MA (2008) Functional coupling of TRPV4 cationic channel and large conductance, calciumdependent potassium channel in human bronchial epithelial cell lines. Pflugers Arch 457:149-159.

Garcia-Elias A, Lorenzo IM, Vicente R, Valverde MA (2008) IP3 receptor binds to and sensitizes TRPV4 channel to osmotic stimuli via a calmodulin-binding site. J Biol Chem 283:31284-31288.

Gottlieb P, Folgering J, Maroto R, Raso A, Wood TG, Kurosky A, Bowman C, Bichet D, Patel A, Sachs F, Martinac B, Hamill OP, Honoré E (2008) Revisiting TRPC1 and TRPC6 mechanosensitivity. Pflugers Arch 455:1097-1103.

Grant AD, Cottrell GS, Amadesi S, Trevisani M, Nicoletti P, Materazzi S, Altier C, Cenac N, Zamponi GW, Bautista-Cruz F, Lopez CB, Joseph EK, Levine JD, Liedtke W, Vanner S, Vergnolle N, Geppetti P, Bunnett NW (2007) Protease-activated receptor 2 sensitizes the transient receptor potential vanilloid 4 ion channel to cause mechanical hyperalgesia in mice. J Physiol 578:715-733.

Hamill OP (2006) Twenty odd years of stretch-sensitive channels. Pflugers Arch 453:333-351.

Hamill OP, McBride DW Jr (1996) The pharmacology of mechanogated membrane ion channels. Pharmacol Rev 48:231-252.
Handwerker HO, Kilo S, Reeh PW (1991) Unresponsive afferent nerve fibres in the sural nerve of the rat. J Physiol 435:229-242.

Hisatsune C, Kuroda Y, Nakamura K, Inoue T, Nakamura T, Michikawa T, Mizutani A, Mikoshiba K (2004) Regulation of TRPC6 channel activity by tyrosine phosphorylation. J Biol Chem 279:18887-18894.

Houck CS, Khodorova A, Reale AM, Strichartz GR, Davar G (2004) Sensory fibers resistant to the actions of tetrodotoxin mediate nocifensive responses to local administration of endothelin-1 in rats. Pain 110:719-726.

Hu J, Lewin GR (2006) Mechanosensitive currents in the neurites of cultured mouse sensory neurones. J Physiol 577:815-828.

Ingber DE (1997) Tensegrity: the architectural basis of cellular mechanotransduction. Annu Rev Physiol 59:575-599.

Ingber DE (2003a) Mechanosensation through integrins: cells act locally but think globally. Proc Natl Acad Sci U S A 100:1472-1474.

Ingber DE (2003b) Tensegrity II. How structural networks influence cellular information processing networks. J Cell Sci 116:1397-1408.

Kim EY, Alvarez-Baron CP, Dryer SE (2009) Canonical transient receptor potential channel (TRPC) 3 and TRPC6 associate with large-conductance $\mathrm{Ca}^{2+}$-activated $\mathrm{K}^{+}$(BKCa) channels: role in BKCa trafficking to the surface of cultured podocytes. Mol Pharmacol 75:466-477.

Kim SJ, Kim YS, Yuan JP, Petralia RS, Worley PF, Linden DJ (2003) Activation of the TRPC1 cation channel by metabotropic glutamate receptor mGluR1. Nature 426:285-291.

Köttgen M, Buchholz B, Garcia-Gonzalez MA, Kotsis F, Fu X, Doerken M, Boehlke C, Steffl D, Tauber R, Wegierski T, Nitschke R, Suzuki M, Kramer-Zucker A, Germino GG, Watnick T, Prenen J, Nilius B, Kuehn EW, Walz G (2008) TRPP2 and TRPV4 form a polymodal sensory channel complex. J Cell Biol 182:437-447.

Kress M, Karasek J, Ferrer-Montiel AV, Scherbakov N, Haberberger RV (2008) TRPC channels and diacylglycerol dependent calcium signaling in rat sensory neurons. Histochem Cell Biol 130:655-667.

Liedtke W, Friedman JM (2003) Abnormal osmotic regulation in trpv4-/mice. Proc Natl Acad Sci U S A 100:13698-13703.

Liu S, Qu MH, Ren W, Hu HZ, Gao N, Wang GD, Wang XY, Fei G, Zuo F, Xia Y, Wood JD (2008) Differential expression of canonical (classical) transient receptor potential channels in guinea pig enteric nervous system. J Comp Neurol 511:847-862.

Lockwich TP, Liu X, Singh BB, Jadlowiec J, Weiland S, Ambudkar IS (2000) Assembly of Trp1 in a signaling complex associated with caveolinscaffolding lipid raft domains. J Biol Chem 275:11934-11942.

Lumpkin EA, Caterina MJ (2007) Mechanisms of sensory transduction in the skin. Nature 445:858-865.

Maroto R, Raso A, Wood TG, Kurosky A, Martinac B, Hamill OP (2005) TRPC1 forms the stretch-activated cation channel in vertebrate cells. Nat Cell Biol 7:179-185.

McCarter GC, Reichling DB, Levine JD (1999) Mechanical transduction by rat dorsal root ganglion neurons in vitro. Neurosci Lett 273:179-182.

Mederos y Schnitzler M, Storch U, Meibers S, Nurwakagari P, Breit A, Essin K, Gollasch M, Gudermann T (2008) G(q)-coupled receptors as mechanosensors mediating myogenic vasoconstriction. EMBO J 27:3092-3103.

Meyers JR, MacDonald RB, Duggan A, Lenzi D, Standaert DG, Corwin JT, Corey DP (2003) Lighting up the senses: FM1-43 loading of sensory cells through nonselective ion channels. J Neurosci 23:4054-4065.

Millan MJ (1999) The induction of pain: an integrative review. Prog Neurobiol 57:1-164.

Park SP, Kim BM, Koo JY, Cho H, Lee CH, Kim M, Na HS, Oh U (2008) A tarantula spider toxin, GsMTx4, reduces mechanical and neuropathic pain. Pain 137:208-217.

Rychkov G, Barritt GJ (2007) TRPC1 Ca(2+)-permeable channels in animal cells. Handb Exp Pharmacol 2007:23-52.

Sharif-Naeini R, Dedman A, Folgering JH, Duprat F, Patel A, Nilius B, Honoré E (2008) TRP channels and mechanosensory transduction: insights into the arterial myogenic response. Pflugers Arch 456:529-540.

Souza AL, Moreira FA, Almeida KR, Bertollo CM, Costa KA, Coelho MM (2002) In vivo evidence for a role of protein kinase $C$ in peripheral nociceptive processing. Br J Pharmacol 135:239-247.

Spassova MA, Hewavitharana T, Xu W, Soboloff J, Gill DL (2006) A common mechanism underlies stretch activation and receptor activation of TRPC6 channels. Proc Natl Acad Sci U S A 103:16586-16591.

Strotmann R, Harteneck C, Nunnenmacher K, Schultz G, Plant TD (2000) 
OTRPC4, a nonselective cation channel that confers sensitivity to extracellular osmolarity. Nat Cell Biol 2:695-702.

Strübing C, Krapivinsky G, Krapivinsky L, Clapham DE (2003) Formation of novel TRPC channels by complex subunit interactions in embryonic brain. J Biol Chem 278:39014-39019.

Suchyna TM, Johnson JH, Hamer K, Leykam JF, Gage DA, Clemo HF, Baumgarten CM, Sachs F (2000) Identification of a peptide toxin from Grammostola spatulata spider venom that blocks cation-selective stretchactivated channels. J Gen Physiol 115:583-598.

Summer GJ, Puntillo KA, Miaskowski C, Dina OA, Green PG, Levine JD (2006) TrkA and PKC-epsilon in thermal burn-induced mechanical hyperalgesia in the rat. J Pain 7:884-891.

Summer GJ, Dina OA, Levine JD (2007) Enhanced inflammatory hyperalgesia after recovery from burn injury. Burns 33:1021-1026.

Summer GJ, Romero-Sandoval EA, Bogen O, Dina OA, Khasar SG, Levine JD (2008) Proinflammatory cytokines mediating burn-injury pain. Pain 135:98-107.

Suzuki M, Mizuno A, Kodaira K, Imai M (2003) Impaired pressure sensation in mice lacking TRPV4. J Biol Chem 278:22664-22668.

Vriens J, Owsianik G, Janssens A, Voets T, Nilius B (2007) Determinants of
4 alpha-phorbol sensitivity in transmembrane domains 3 and 4 of the cation channel TRPV4. J Biol Chem 282:12796-12803.

Watanabe H, Davis JB, Smart D, Jerman JC, Smith GD, Hayes P, Vriens J, Cairns W, Wissenbach U, Prenen J, Flockerzi V, Droogmans G, Benham CD, Nilius B (2002) Activation of TRPV4 channels (hVRL-2/mTRP12) by phorbol derivatives. J Biol Chem 277:13569-13577.

Willis WD Jr (1985) The pain system. The neural basis of nociceptive transmission in the mammalian nervous system. Pain Headache 8:1-346.

Yuan JP, Kiselyov K, Shin DM, Chen J, Shcheynikov N, Kang SH, Dehoff MH, Schwarz MK, Seeburg PH, Muallem S, Worley PF (2003) Homer binds TRPC family channels and is required for gating of TRPC1 by IP3 receptors. Cell 114:777-789.

Zhang XY, Bai ZT, Chai ZF, Zhang JW, Liu Y, Ji YH (2003) Suppressive effects of BmK IT2 on nociceptive behavior and c-Fos expression in spinal cord induced by formalin. J Neurosci Res 74:167-173.

Zheng JH, Chen J (2001) Differential roles of spinal neurokinin 1/2 receptors in development of persistent spontaneous nociception and hyperalgesia induced by subcutaneous bee venom injection in the conscious rat. Neuropeptides 35:32-44. 